OPEN ACCESS

Edited by:

Maryam Mehrpour,

Université Paris Descartes,

France

Reviewed by:

Richard Possemato,

NYU Grossman School of Medicine,

United States

Keith R. Laderoute,

Consultant, Redwood City, CA

United States

*Correspondence:

Wenzhou Zhang

hnzzzwzx@sina.com

Xiaoman L

lixm@njucm.edu.cn

Lufeng Zheng

zhl@@cpu.edu.cn

${ }^{\dagger}$ These authors have contributed equally to this work

Specialty section:

This article was submitted to Molecular and Cellular Oncology,

a section of the journal

Frontiers in Oncology

Received: 17 September 2021

Accepted: 15 October 2021

Published: 10 November 2021

Citation:

Guo Q, Li L, Hou S, Yuan Z, Li C, Zhang W, Zheng $L$ and LiX (2021) The Role of Iron in Cancer Progression.

Front. Oncol. 11:778492.

doi: 10.3389/fonc.2021.778492

\section{The Role of Iron in Cancer Progression}

\author{
Qianqian Guo ${ }^{1 \dagger}$, Liwen $\mathrm{Li}^{3 \dagger}$, Shanshan $\mathrm{Hou}^{4}$, Ziqiao Yuan ${ }^{5}$, Chenhui $\mathrm{Li}^{3}$, \\ Wenzhou Zhang ${ }^{1 *}$, Lufeng Zheng ${ }^{3 *}$ and Xiaoman $\mathrm{Li}^{2 *}$ \\ 1 Department of Pharmacy, Affiliated Cancer Hospital of Zhengzhou University, Henan Cancer Hospital, Zhengzhou, China, \\ 2 Jiangsu Key Laboratory for Pharmacology and Safety Evaluation of Chinese Materia Medica, School of Pharmacy, Nanjing \\ University of Chinese Medicine, Nanjing, China, ${ }^{3}$ School of Life Science and Technology, Jiangsu Key Laboratory of \\ Carcinogenesis and Intervention, School of Basic Medicine and Clinical Pharmacy, China Pharmaceutical University, Nanjing, \\ China, ${ }^{4}$ Department of Pharmacy, Zhejiang Pharmaceutical College, Ningbo, China, ${ }^{5}$ School of Pharmaceutical Sciences, \\ Zhengzhou University, Zhengzhou, China
}

Iron is an essential trace element for the human body, and its deficiency or excess can induce a variety of biological processes. Plenty of evidences have shown that iron metabolism is closely related to the occurrence and development of tumors. In addition, iron plays an important role in cell death, which is very important for the development of potential strategies for tumor treatment. Here, we reviewed the latest research about iron metabolism disorders in various types of tumors, the functions and properties of iron in ferroptosis and ferritinophagy, and new opportunities for iron-based on treatment methods for tumors, providing more information regarding the prevention and treatment of tumors.

Keywords: iron, cancer, ferroptosis, ferritinophagy, prognostic, therapy

\section{INTRODUCTION}

Iron $(\mathrm{Fe})$ is one of the most abundant elements in the earth's crust (1). The oxidation-reduction reaction mainly involves electron transfer between two chemicals. Iron, as a transition-metal, can exhibit a wide range of oxidation states, which makes it a multifunctional participant in redox reactions. Therefore, iron is an indispensable trace element to maintain life (2). This element plays a vital role in various cellular processes, such as cellular respiration (e.g., cytochrome c oxidase, ferredoxin, cytochrome, and Rieske protein), energy metabolism (e.g., aconitase, citrate synthase, succinate dehydrogenase, and isocitrate dehydrogenase), DNA replication, DNA synthesis and nucleic acid repair (e.g., the catalytic subunit of replicative DNA polymerases, DNA helicase and ribonucleotide reductase), and iron-dependent signaling $(3,4)$. Iron is also used in the synthesis of heme and iron-sulfur clusters (ISC), which are incorporated into proteins that carry out the citric acid cycle, oxidative phosphorylation, and many other essential functions (5, 6). However, although

\footnotetext{
Abbreviations: DCYTB, duodenal cytochrome B; DMT1, divalent metal transporter 1; HO-1, heme oxygenase-1; FPN1, ferroportin; HEPH, hephaestin; TF, transferrin; TFR1, transferrin receptor; STEAP3, six-transmembrane prostate epithelial antigen 3; LIP, labile iron pool; IRPs, iron-responsive element binding proteins; IRE, iron responsive element; mRNA, messenger RNA; BMP6, bone morphogenetic protein; ROS, reactive oxygen species; DFO, Desferoxamine; IREB2, Iron responsive element binding protein 2 gene; ISC, iron-sulfur cluster; GSSG, glutathione disulphide; GSH, glutathione; L-OHs, lipid alcohols; L-OOHs, lipid hydroperoxides; PUFA, polyunsaturated fatty acids; GPX4, glutathione peroxidase 4; NCOA4, nuclear receptor coactivator 4; FTH1, ferritin heavy chain 1; FTL, ferritin light chain.
} 
iron is essential for the normal physiological function of the human body, it may also be toxic in that it generates a large number of free radicals in the presence of hydrogen peroxide (7). For example, in the well-known Fenton reaction, ferrous iron $\left(\mathrm{Fe}^{2+}\right)$ reacts with hydrogen peroxide to be oxidized to ferric iron $\left(\mathrm{Fe}^{3+}\right)$ while generating hydroxyl radicals. When superoxide is present, the $\mathrm{Fe}^{3+}$ produced by the Fenton reaction can be reduced to $\mathrm{Fe}^{2+}$, and then the Fenton reaction will proceed again, which called Haber-Weiss reaction (8). Both the Fenton reaction and Haber-Weiss reaction can produce a large number of hydroxyl radicals. Hydroxyl radical is one of the most important oxidant found in human body, which can lead to peroxidation and apoptosis by attacking protein, lipids, nucleic acids, and carbohydrates (9, 10). In addition, excessive free radicals in the human body can lead to cell and tissue organ damage, and these processes are closely related to tumorigenesis.

Therefore, iron is not only an essential nutrient element, but also potentially toxic. Both aspects play important roles in the occurrence and development of tumors. This article mainly discusses iron metabolism disorders in tumors, ferroptosis, ferritinophagy, and the role of iron in tumor treatment.

\section{PHYSIOLOGICAL MECHANISMS OF IRON REGULATION}

Homeostasis of iron metabolism is a physiological process that needs to be strictly controlled. Iron is mainly present in the oxidized state $\left(\mathrm{Fe}^{3+}\right)$ and is divided into dietary iron and environmental iron. Dietary iron primarily exists as either nonheme bound iron or heme (11). Heme iron has a higher absorption rather than nonheme bound iron. Iron in the diet is mainly reduced to $\mathrm{Fe}^{2+}$ in the duodenum by duodenal cytochrome B (DCYTB) and absorbed into intestinal epithelial cells under the synergistic effect of divalent metal transporter 1 (DMT1) (1). Heme iron is also absorbed by intestinal cells through an unknown mechanism and is metabolized by heme oxygenase-1 (HO-1) to release $\mathrm{Fe}^{2+}$. Heme iron is absorbed by intestinal cells through an unknown mechanism and degraded by heme oxygenase-1 (HO-1), releasing $\mathrm{Fe}^{2+}$, which is transported out of the cells by the iron efflux pump ferroportin (FPN1) on the basal side of the intestinal epithelial cells, and consequently oxidized into $\mathrm{Fe}^{3+}$ by hephaestin (HEPH) (1); then $\mathrm{Fe}^{3+}$ binds to transferrin (TF) and enters the circulation through the portal vein. Each transferrin in blood can bind two $\mathrm{Fe}^{3+}$ to form TF- $\left[\mathrm{Fe}^{3+}\right]_{2}$ complex, which binds to transferrin receptor (TFR1) on the cell surface and is absorbed into cells to form endosome (12). Subsequently, it is reduced to $\mathrm{Fe}^{2+}$ by sixtransmembrane prostate epithelial antigen 3 (STEAP3), which is then transported into cytoplasm by DMT1 to exert physiological functions or constitute the cytoplasmic labile iron pool (LIP) (1, 13). LIP can be taken up by non-hematopoietic cells causing parenchymal iron deposition which can result in free radical damage (14) (Figure 1).

Intracellular iron homeostasis is mainly regulated through an iron-dependent protein network including iron-responsive

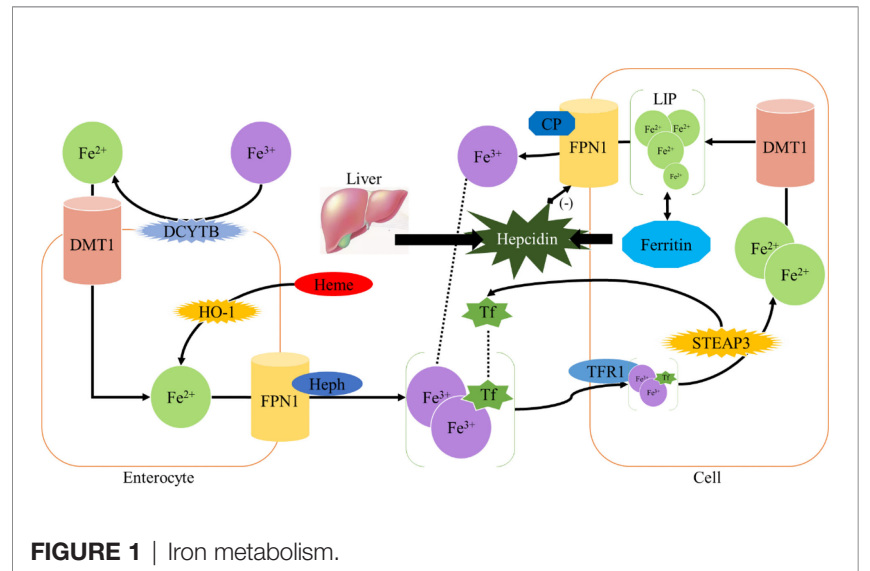

element binding proteins (IRPs), in which both IRP1 and IRP2 are important components (15). It is noteworthy that thioredoxin family proteins are important mediators in iron metabolism since these proteins regulate the expression of IRPs (5). To ensure iron homeostasis, IRP binds to the corresponding iron responsive element (IRE) on the untranslated region of messenger RNA encoding the protein essential for cellular iron regulation (TFR1, DMT1), thereby participating in iron uptake (TFR1), storage (FT), redistribution, and efflux (FPN1) (2). In the absence of intracellular iron, IRP can inhibit the translation of FPN1 and FT, but increase the protein synthesis of TFR1. In contrast, when the intracellular iron is abundant, the synthesis of FPN1 and FT is increased due to the instability of IRP, while the degradation of TFR1 is promoted (16). IRP1/2 are key iron regulators for the maintenance of cellular iron homeostasis. IRP1 is cytosolic aconitase, an enzyme containing a [4Fe-4S] cluster. When absence of intracellular iron, there is insufficient iron for $\mathrm{Fe}-\mathrm{S}$ biogenesis leaving an incomplete [3Fe-4S] cluster. The enzymatic activity of aconitase is lost and this protein then initiates its IRP activity, as IRP1. When the protein contains the $[3 \mathrm{Fe}-4 \mathrm{~S}]$ cluster it can bind to IREs $(17,18)$. Through these mechanisms, IRP can not only meet the metabolic needs of cellular iron, but also minimize the toxic effects of excessive iron (Figure 2).

The excess iron is mainly stored in liver, which also acts as an iron-sensing organ and controls systemic iron through the secretion of the peptide hormone hepcidin (19). Hepcidin is a 25-amino acid short peptide produced by the liver and encoded by the HAMP (20). FPN1, which is mainly expressed in the cell membrane and cytoplasm, is the only known 571-amino acid transmembrane protein in vertebrates that transports iron from intracellular to extracellular (21). When the intracellular or circulating iron level is relatively high, Hepcidin is generated from hepatocytes and secreted into the circulation system through a bone morphogenetic protein (BMP6) - mediated pathway $(1,22)$. Hepcidin can trigger the internalization and subsequent lysosomal degradation of FPN1 via binding to the basal side of the intestinal epithelium and to the FPN1 on the macrophage surface, preventing iron absorption from the digestive tract and iron circulation from the body, respectively (23). Conversely, the reduction of iron modulators causes FPN1 


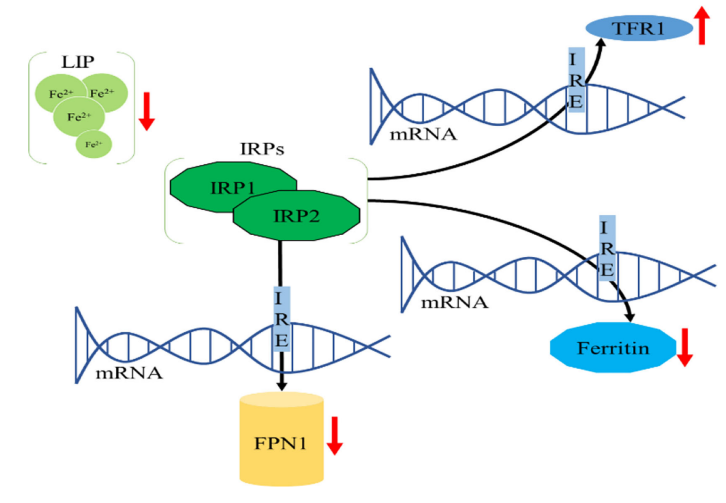

FIGURE 2 | Cellular iron metabolism. (A) When the cellular iron levels are low, IRPs bind to the 5' IREs of FPN1 and FT mRNA, resulting in translation repression. Additionally, IRPs bind to 3' IREs in TFR1 mRNA to stabilize the DMT1 mRNA and increase TFR1 protein synthesis. These two effects can increase intracellular iron. (B) When the cellular iron levels are high, IRP1 converts to cytosolic aconitase and IRP2 is degraded, preventing IRP1 and IRP2 from binding to the IREs of these mRNAs. This permits unimpeded translation of FPN1 and FT and fosters degradation of DMT1 and TFR1 $\mathrm{mRNA}$, leading to decreased cytosolic iron.

to absorb iron and increases systemic iron levels (24) (Figure 1). Therefore, FPN1 and ferritin are essential for maintaining iron homeostasis in the body.

\section{IRON METABOLISM IN CANCER}

A large number of studies have shown that abnormal iron homeostasis is one of the markers of cancer (Table 1). As the metabolism and proliferation rate of tumor cells are generally higher than that of normal cells, so their demand for iron is also significantly higher than that of normal cells, this leading to the exceeding oxidative stress; however, tumor cells can exert a concomitant upregulation of antioxidant defenses for survival, such as activating antioxidant transcription factors and promoting the expression of various antioxidant genes (57). Conversely, since tumor cells are strongly dependent on iron for their growth/proliferation, they are more sensitive to iron depletion than normal cells. This imbalance in cancer is mainly manifested as increased iron metabolism, iron affinity, iron input, and inhibition of its output, thereby completing iron accumulation.

\section{Iron Uptake TFR1}

As early as 1980, studies have shown that TFR1 is significantly up-regulated in breast cancer (27). Subsequent studies have confirmed that TFR1 is highly expressed in various cancers such as glioma, leukemia, breast cancer, and ovarian cancer $(25,26,28,29)$. Recent studies have shown that Beclin 1 inhibits the proliferation of breast cancer cells by regulating the endocytosis transport and degradation of EGFR and TFR1 (58). Bajbouj et al. found that estrogen inhibited the synthesis of ferritin and enhanced the efflux of intracellular iron. Meanwhile, combining with doxorubicin, estrogen significantly reduced TFR1 expression and enhanced the sensitivity of breast cancer cells to doxorubicin (59). Using Gene Expression Profiling Interactive Analysis (GEPIA) database analysis, Xiao et al. found that TFR1 was significantly up-regulated in hepatocellular carcinoma tissues and hepatocellular carcinoma stem cells (31), and knocking down TFR1 reduced iron accumulation, reactive oxygen species (ROS) accumulation induced by erastin, and maintained mitochondrial function, thereby inhibiting tumor development (31). Additionally, a higher expression of TFR1 is closely related to the activation of ERK signaling pathway in thyroid cancer, leading to the disorder of genes involved in abnormal accumulation of intracellular free iron and drug resistance (33).

Other studies have shown that targeting TFR1 can suppress tumor progression, such as MiR-107 can inhibit the proliferation, migration, and invasion of SW620 cells by targeting TFR1 (34); MiR-148a could reduce the proliferation of liver cancer cells by targeting TFR1 (32); EGFR regulates cellular iron homeostasis by regulating the redistribution of TFR1, and promotes the development of non-small cell lung cancer (60). Similarly, knock-down of ST6GALNACIII can down-regulate TFR1 and delay A549 cell proliferation (61). And Hui et al. found that TFR1 level was significantly upregulated in non-small-cell lung cancer (NSCLC) by Fusaricide, which is a novel iron chelating agent and can efficiently inhibit the proliferation of a variety of human NSCLC cell lines (62). In addition, TFR1 is highly expressed in activated lymphocytes and malignant cells, and TFR1 antibody ch128.1/IgG1 effectively inhibits the activation, growth, and immortalization of $E B V^{+}$human $B$ cells as well as the development of these cells into lymphoma-like tumors in immunodeficient mice (63). Chlorogenic acid inhibits the growth of pancreatic ductal adenocarcinoma cells by targeting the c-Myc-TFR1 axis, perhaps chlorogenic acid is a promising compound for pancreatic ductal adenocarcinoma therapy (64). However, although TFR 1 is highly expressed in colorectal cancer tissues, the authors demonstrated that down-regulation of TFR1 promoted rectal cell migration and invasion through the JAK/ STAT pathway (35). These results demonstrate that TFR1 might hold tumor-specific roles.

\section{DMT1}

DMT1 is highly expressed in various cancers, such as colorectal cancer and ovarian cancer $(30,38)$. Q. Wang et al. found that DMT1, TFR1, and ferritin were highly expressed in ovarian cancer cell spheres, and overexpression of DMT1 promoted the progression of ovarian tumor (30). In SDHB-Mutated Pheochromocytoma, iron accumulation caused by significant up-regulation of iron transport-related proteins, such as DMT1, TF, TFR2, can increase oxidative stress to some extent (37). In colorectal cancer, DMT1 can be induced through hypoxia-inducible factor $2 \alpha$-dependent transcription (38). However, in hepatocellular carcinoma, patients with a lower expression of DMT1 displayed a worse disease-free survival, this effect was more significant in patients with advanced 
TABLE 1 | Iron regulators in cancer.

\begin{tabular}{|c|c|c|c|}
\hline Altered Player & Cancer & Regulation & Ref. \\
\hline TFR1 & glioblastoma & up & (25) \\
\hline TFR1 & leukemia & up & (26) \\
\hline TFR1 & breast cancer & up & $(27,28)$ \\
\hline TFR1 & ovarian cancer & up & $(29,30)$ \\
\hline TFR1 & hepatic carcinoma & up & $(31,32)$ \\
\hline TFR1 & thyroid cancer & up & (33) \\
\hline TFR1 & colorectal cancer & up & $(34-36)$ \\
\hline DMT1 & ovarian cancer & up & $(30)$ \\
\hline DMT1 & pheochromocytoma & up & (37) \\
\hline DMT1 & colorectal cancer & up & (38) \\
\hline STEAP3 & glioblastoma & up & (39) \\
\hline STEAP3 & kidney renal clear cell carcinoma & up & (39) \\
\hline STEAP3 & Liver Hepatocellular Carcinoma & down & (28) \\
\hline IRP2 & colorectal cancer & up & (36) \\
\hline IREB2 & lung cancer & up & $(40,41)$ \\
\hline FPN & pancreatic cancer & down & $(42)$ \\
\hline FPN & multiple myeloma & down & (43) \\
\hline FPN & thyroid cancer & down & (44) \\
\hline Hepcidin & breast cancer & up & (45) \\
\hline Hepcidin & colorectal cancer & up & $(46)$ \\
\hline Hepcidin & prostate cancer & up & $(47,48)$ \\
\hline Hepcidin & thyroid cancer & up & $(44)$ \\
\hline Hepcidin & breast cancer & up & (49) \\
\hline Hepcidin & multiple myeloma & up & $(50,51)$ \\
\hline Hepcidin & non-Hodgkin's lymphoma & up & $(52)$ \\
\hline Hepcidin & UUTUC & up & (53) \\
\hline Hepcidin & Renal Cell Carcinoma & up & (53) \\
\hline Hepcidin & hepatocellular carcinoma & down & (54) \\
\hline HAMP & hepatocellular carcinoma & down & (55) \\
\hline HAMP & cholangiocarcinoma & down & (56) \\
\hline
\end{tabular}

hepatocellular carcinoma (65). Notably, the carcinogenic activity of DMT1 is tightly correlated with its iron-transport activity, which is characterized by the evidence that tumor in DMT1knocked out mice was weakened when being fed with a high-iron diet. Additionally, Desferoxamine (DFO) increases iron concentration by up-regulating the expression of DMT1 and TFR1, thereby promoting the migration of breast cancer cells (66). Similarly, the latest research by Chen et al. has revealed that the up-regulation of DMT1 and TFR1 is related to the activation of the IL-6/PI3K/AKT signaling pathway in triple-negative breast cancer cells, and enhance iron absorption (67). Consistently, targeting DMT1 can be used to potentially suppress tumor progression. For example, DMT1 inhibitors can selectively target stem cells in primary cancer cells and circulating tumor cells to inhibit the occurrence and development of tumors (68); Propofol, which is widely used in clinical practice for intraoperative general anesthesia and postoperative sedation, regulates DMT1 expression by modifying Ca2+-permeable $\alpha$-amino-3-hydroxyl-5methylisoxazole-4-propionic acid receptors (CPARs), thereby inhibiting tumor oxidative stress and glioma tumor growth (69).

\section{STEAPs}

The expression STEAPs also exhibits an upregulation in tumors and they can promote tumor cell proliferation as well as suppress apoptosis (70-73), for example, STEAP3 promotes glioma cell proliferation, invasion, and spheroid formation by inducing mesenchymal transformation, promoting TFR1 expression, and activating the STAT3-FoxM1 axis (39); In colorectal cancer, STEAP3 expression is significantly higher in tumor than that in colonic mucosa (74). However, they recently found that silencing lncRNA STEAP3-AS1(the antisense transcript of STEAP3) inhibited the proliferation, migration, and arrested colon cancer cells at the G0-G1 phase cancer cells through upregulation STEAP3 (75). Additionally, Z. Wang et al. found that among Burkitt's lymphoma, the rarely studied iron reductase CYB561A3 was essential for Burkitt proliferation, but lymphoblastoid B cells with the EBV latency III program depended on STEAP3 iron reductase instead (76). Similarly, STEAP2 accelerates prostate cancer progression by promoting proliferation, migration, and invasion by regulating the transcriptional profiles of some genes involved in metastasis. And Burnell et al. proved that reducing the expression of STEAP2 inhibited the proliferation, migration, and invasion in prostate cancer cells (77).

\section{Intracellular Iron Regulation}

IRP1 or IRP2 can increase intracellular iron and abnormal activation of them is closely related to many cancers. Compared with normal colonic mucosa, IRP2 is overexpressed in colorectal cancer, and is positively correlated with TFR1 expression. In addition, the expression of IRP2 is associated with mutations of BRAF, which primarily occur in the early stages of colorectal cancer and are often associated with poor 
prognosis (36). IRP1 and IRP2 are strictly regulated in tumors, and preferably modulate tumor progression in an iron-engaged signaling pathways, such as IRP2 is regulated by ubiquitin ligase FBXL5, which mediated IRP2 ubiquitination and degradation under the condition of sufficient iron; Dysregulation of FBXL5 has been associated with a poor prognosis of human hepatocellular carcinoma (78); A recent study demonstrated that FBXL5 is stabilized and increases iron levels when iron is at low levels, facilitating the production of [2Fe2S] cluster, which can serve as an FBXL5 cofactor, and by incorporating into the Cterminal LRR domain of FBXL5, and only when oxygen level is high enough to maintain the [2Fe2S] cluster in its oxidized $[2 \mathrm{Fe} 2 \mathrm{~S}]^{2+}$ state could the $\mathrm{SCF}^{\mathrm{FBXL} 5} \mathrm{E} 3$ ligase recruits IRP2 as a substrate for polyubiquination and degradation. This IRP2FBXL5 interaction might help release IRP2 from IREs to alter the translation of iron metabolism genes (79). IRP2 is also regulated by the deubiquitinating enzyme OTUD1, which promotes TFR1-mediated iron transport via deubiquitinating and stabilizing IRP2, leading to increased ROS production, and the downregulation of OTUD1 has been found to be highly correlated with poor prognosis of colorectal cancer (80); A study linking a clinical trial of JBR.10 $(n=131)$ with a sample of patients from the University of Toronto Health Network ( $\mathrm{n}=$ 181) indicated that the effect of the $15 q 25$ mutation on lung cancer risk was associated with increased expression of IREB2 (40); Another large-scale case-control study confirmed that the miRNA binding site SNP rs1062980 in the IREB2 3' UTR might potentially alter IREB2 expression to reduce the risk of lung cancer by regulating the binding of miR-29a (41). Additionally, the specific cause of dysregulation of IRP1/2 may be related to inhibition of TAp63 and activation of MDM2 (81, 82).Notably, chemotherapy and targeted-therapy may work together to disrupt IRP-mediated iron regulation, like Horniblow et al. found that the MEK inhibitor trimetinib consistently inhibited IRP2 expression in four colorectal cell lines, resulting in decreased TFR1 expression and increased ferritin expression (83); Miyazawa et al. found that cisplatin bound to Cys512 and Cys516 of human IRP2 and destroyed its function, based on which DFO combined with cisplatin resulted in increased iron consumption and reduced tumor growth in a mouse xenograft model of colonic adenocarcinoma (84); Yao et al. confirmed that erastin and RSL3 increased the expression of IRP1 and IRP2, knockdown of which conversely inhibited erastin- or RSL3induce cell death, and IRP2 could enhance the promoting effect of IRP1 in melanoma cells (85).

\section{Iron Efflux in Cancer \\ Ferroportin}

FPN1, the only iron export protein, is involved in the regulation of intracellular iron concentration, and its abnormal downregulation is also observed in most tumors (29, 47, 86, 87). The suppressive roles of FPN1 have been established in tumors, such as in prostate cancer cell lines, a low FPN1 level caused by up-regulation of ferritin promoted proliferation, migration, and apoptotic resistance, and overexpression of FPN1 induced p53 and autophagy, and reduced tumor growth in vivo (42); Overexpression of FPN1 decreased proliferation, colony formation, and tumor growth, as well as liver metastasis in breast cancer (88). In consistent, various studies have indicated that FPN1 regulates tumor progression via destroying iron homeostasis, as a study demonstrated that cadmium (Cd)-induced MDA-MB-231 cell proliferation, EMT, and migration were caused by inhibiting FPN1 expression and associated with destruction of iron homeostasis (89), and hepcidin secreted by thyroid cancer cells could decrease FPN1 and retain intracellular iron, thereby promoting cancer proliferation (44); another study showed that FPN1-mediated iron metabolism might play a role in chemosensitivity and treatment outcome of acute myeloid leukemia (90); Similarly, overexpression of ZNF217 promoted prostate cancer growth by inhibiting FPN1-conducted iron efflux (91); Xue et al. found that $\mathrm{Nrf2}$, a transcription factor of FPN1, inhibited the proliferation and metastasis of prostate cancer cells by up-regulating FPN1, which was also related to the reduction of intracellular ferritin content (92), and Nrf2 was able to inhibit myeloma cell proliferation by promoting FPN1 transcription (43). Additionally, Geng et al. demonstrated that knockdown of FPN1 accelerated erastin-induced suppression of neuroblastoma by increasing the accumulation of irondependent lipid ROS, and FPN1 inhibitors might provide a new approach for the chemosensitization of neuroblastoma (93); Tang et al. verified that USP35 could maintain the stability of its protein by deubiquitinating FPN1, and reduce the iron disorder triggered by erastin/RSL3, thereby promoting lung cancer cell growth and tumor progression. Meanwhile, knockdown of USP35 enhanced the sensitivity of lung cancer cells to cisplatin and paclitaxel by targeting FPN1 in lung cancer (94). However, a study showed that matrix/macrophage expression of Lcn-2 was associated with tumor onset, lung metastasis, and recurrence, whereas FPN1 was not by analyzing the expression profiles of lipocalin-2 ( Lcn-2) and FPN1 through a model of T-oncogene (PyMT) breast cancer in spontaneous polymerases and mining publicly available TCGA and GEO database from gene expression synthesis (95). Macrophages provide iron for the microenvironment of breast tumors via forcing secretion of Lcn-2-bound iron and increasing expression of FPN1 (95). Nevertheless, the reason why the matrix/macrophage expression of FPN1 is not associated with tumor onset, lung metastasis, and recurrence requires further investigation.

\section{Hepcidin}

Disorders of iron modulators in cancer cause changes in iron homeostasis. Hepcidin, a negative regulator of FPN1, is significantly up-regulated in various tumors, such as breast, colorectal, and prostate cancer (45-47). And numerous studies have shown the promoting effects of hepcidin on tumor progression, for example, Schwartz et al. found that compared with wild-type littermates, mice lacking hepcidin in colonic tumor epithelium significantly reduced the number, tumor burden and size in the sporadic model of colorectal cancer, whereas lacking of FPN1 led to intracellular iron accumulation and promoted tumor occurrence (96); Lopes et al. found that acute myeloid leukemia had a unique iron component, 
mainly manifested as a low expression of transferrin, and high expression of ferritin and hepcidin; notably, these characteristics were not related to inflammation or blood transfusion (50); Zhao et al. confirmed that hepcidin enhanced the proliferation, migration, and anti-apoptotic capabilities of prostate cancer cells by reducing the expression of FPN1 and increasing intracellular iron level (48); Additionally, Zhou et al. identified that the synthesis of hepcidin is regulated by SOSTDC1, the BMP4/7 antagonist, providing a new mechanism for cellular iron dysfunction via the E4BP4/G9a/ SOSTDC1/hepcidin pathway in the thyroid gland, which can inhibit the secretion of hepcidin and proliferation of thyroid cancer cells (44).

In addition to the iron modulators synthesized by cancer cells, systemic iron disorders in cancer patients can also be observed $(88,97,98)$. Serum hepcidin is elevated in many cancer patients, including prostate cancer, breast cancer, multiple myeloma, and non-Hodgkin's lymphoma (48, 49, 51, 52).A study of 456 cases of primary gastric adenocarcinoma and 900 matched controls with an average of 11 years of followup showed a significant negative correlation between gastric cancer risk and serum hepcidin level, which was mainly caused by ferritin level (99). Additionally, treatment with morotinib was found to decrease serum hepcidin and improve iron metabolism and erythropoiesis in a Phase 2 clinical trial of morotinib for bone marrow fibrosis (100). Similarly, a retrospective study, including 38 patients suffering from upper urinary tract urothelial carcinoma (UUTUC), 94 patients Suffering from Renal Cell Carcinoma (RCC), and 21 patients without infections or cancer, showed that serum hepcidin level was significant increased compared to sera of controls in patients with UUTUC and RCC, and high serum hepcidin was associated with cancer recurrence and metastasis (53).

Interestingly, liver cancers showed a drastic reduction of hepcidin expression compared to benign liver tissues (101, 102). Furthermore, in patients of the White race with no history of alcohol consumption, down-regulation of hepcidin is associated with rapid cancer progression and poor diseasespecific survival. Hepcidin expression is positively correlated with BMP6/interleukin -6 (IL6) cytokines and cytotoxic immune infiltration in liver cancer tissues (54). In addition, blocking hepcidin with its antagonist furthiomine could moderately reduce sorafenib-induced apoptosis in HepG2 and Huh7 cells (54). In consistent, HAMP, the coding gene for hepcidin is mainly expressed in benign liver tissues but significantly reduced in hepatocellular carcinoma tissues (54, 55). Similarly, Z. Wang et al. found that HAMP was decreased and associated with the chemokine CCL16 in Cholangiocarcinoma (CHOL), the second common malignant tumor in the liver, indicating that HAMP may contribute to the immune activation in CHOL microenvironment (56).

Heme has been indicated to promote cell proliferation in leukemia and lung cancer, and increase $\mathrm{HO}-1$ activity promotes invasion and migration of breast cancer cells (103-105). Upregulation of BMP, which induces cancer cells to secrete hepcidin, has also been observed in various tumors, including breast, prostate, and bladder cancers $(47,106,107)$.

In conclusion, cancer cells usually increase the input of iron and inhibit its output, thereby achieving iron accumulation. However, it is not entirely clear how they respond to the increased instability.

\section{IRON AND CELL DEATH}

\section{Iron and Ferroptosis}

In recent years, non-apoptotic cell death has attracted a widespread attention in tumor therapy, among which ferroptosis is defined as iron-dependent regulatory necrosis caused by membrane damage mediated by massive lipid peroxidation (108-110) (Figure 3). Upon being experienced ferroptosis, cells show unique signs such as cell membrane rupture, cytoplasmic swelling, cytoplasmic organelle swelling, mitochondrial membrane density increase, mitochondrial cristae reduction/disappearance, mitochondrial outer membrane rupture, etc. Ferroptosis can occur through two main pathways: the external or transporter-dependent pathway, and the internal or enzyme-regulated pathway (111). Ferroptosis is caused by the redox imbalance between the production of oxidants and antioxidants, which is driven by the abnormal expression and activity of numerous redox active enzymes that produce or detoxify free radicals and lipid oxidation products (109, 111).

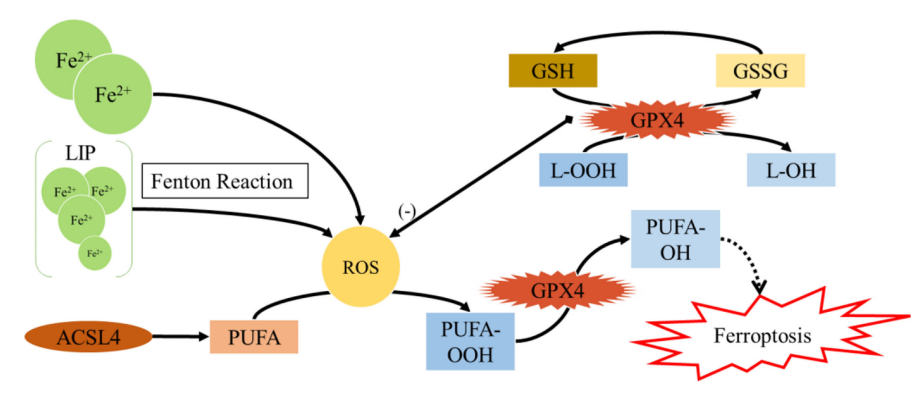

FIGURE 3 | Ferroptosis. 
As the center of metabolism, mitochondria are an important source of ROS in most mammalian cells. Earlier studies showed that mitochondrial-mediated ROS production was not necessary for ferroptosis (108). However, recent studies have shown that mitochondrial-mediated ROS production, DNA stress, and metabolic reprogramming are necessary for lipid peroxidation and induction of ferroptosis (112-114). Readers can obtain a more comprehensive understanding of ferroptosis by referring to other relevant documents $(111,115-118)$. Here we focus on recent research, aiming to clarify the possible novel mechanisms of ferroptosis in cancer.

The previous study suggested that the stability of IRP2 protein was mainly regulated by E3 ubiquitin ligase FBXL5 (78), while Terzi et al. confirmed a new regulatory mechanism of IRP2, which senses the absence of ISC synthesis, and ISC defects could enhance the binding of IRP2 to target mRNA ignoring the changes in IRP1, IRP2, and FBXL5 protein levels. ISC are made up of iron and sulfur ions to form [1Fe-0S], [2Fe$2 \mathrm{~S}],[3 \mathrm{Fe}-4 \mathrm{~S}]$ and $[4 \mathrm{Fe}-4 \mathrm{~S}]$ clusters $(6,119)$. Suppressing ISC synthesis can activate IRP2 and promote ferroptosis sensitivity (120); in line with this finding, insufficient ISC maintenance has been shown to robustly activate the iron-starvation response and trigger ferroptosis (121). Notably, inorganic sulfur is first produced from the cysteine by the cysteine desulfurase NFS1 and ISC is formed on the ISC assembly enzyme (ISCU) with the help of frataxin (FXN) (122), Which was indicated to be localized in the mitochondrial matrix and participated in the biosynthesis of ISC and FXN deficiency accelerates erastin-activated ferroptosis (123). Furthermore, overexpression of ISCU significantly attenuated Dihydroartemisinin-induced ferroptosis by regulating iron metabolism, rescuing the mitochondrial function and increasing the level of GSH (124). Additionally, FXN could activate NFS1 and accelerate a rate-limiting sulfur transfer step of ISC assembly, and suppression of NFS1 make cancer cell be sensitive to ferroptosis $(121,125)$. Besides, Chafe et al. identified a novel synthetic lethal interaction between carbonic anhydrase IX (CAIX) and NFS1 by elucidating the important role of CAIX in redox homeostasis and the prevention of ferroptosis through $\mathrm{pH}$ regulation, which may facilitate researchers to develop new strategies for the treatment of solid tumors (126).

The latest research provides more possibilities that targeting ferroptosis may be a new strategy for tumor treatment. For example, Mao et al. identified a ferroptosis-defensive mechanism mediated by Dihydroorotate dehydrogenase (DHODH) in the mitochondria, which works with mitochondrial glutathione peroxidase 4 (GPX4) to reduce ubiquinone to panthenol, thereby inhibiting ferroptosis in mitochondrial inner membrane, while Brequinar (a DHODH inhibitor) selectively inhibits the proliferation of tumor cells with low GPX4 expression by inducing ferroptosis, thus it can occur synergistically to induce ferroptosis and inhibit the growth of tumor cells with high GPX4 expression by the combined use of Brequinar and sulfasalazine (ferroptosis inducers) (127); Subsequently, Ding et al. demonstrated that DMOCPTL induced ferroptosis and apoptosis primarily through GPX4 ubiquitination in triple-negative breast cancer cells (128); Additionally, D. Chen et al. confirmed that IPLA2 $\beta$ inhibited ferroptosis by cleaving through lipid peroxide for detoxify without depending on GPX4, and that the absence of iPLA2 $\beta$ had no significant effect on the normal development or cell viability of normal tissues, thus, iPLA2 $\beta$ may become a new target for ferroptosis-targeted therapy for tumor (129); Furthermore, X. Wang et al. demonstrated that SOX2 promoted SLC7A11 transcription by binding to SLC7A11 promoter, and oxidation at Cys 265 of SOX2 inhibited its activity and decreased the self-renewal capacity of lung cancer stem cell-like cells. This suggests that oxidation of SOX2 could be a potential target for ferroptosis-targeted treatment for cancer (130). Moreover, as cell density-dependent E-cadherin and Merlin/Neurofibromin (NF2) loss can induce ferroptosis, Bao et al. found that NF2-inactivated meningioma cells were sensitive to Erastin-induced ferroptosis by analyzing 35 meningioma samples (10 NF2 loss and 25 NF2 wildtype), and further confirmed that myoenhancer factor $2 \mathrm{C}(M E F 2 C)$ acted as a promoter of $\mathrm{NF} 2$ and $\mathrm{CDH} 1$, thereby inhibiting ferroptosisrelated lipid peroxidation and meningioma cell death (131). Notably, Kremer et al. indicated that aspartate aminotransferase (GOT1) could damage mitochondrial oxidative phosphorylation and promote catabolism, resulting in the increase of unstable iron pool and susceptibility to ferroptosis, this effect suggests that inhibiting GOT1 could destroy the redox balance and proliferation in pancreatic ductal carcinoma, and establishes a biochemical link between GOT1 and ferroptosis (132). Few studies reported the direct crosstalk between ferroptosis and antitumor immunity, until Wang et al. reported that $\mathrm{CD}^{+} \mathrm{T}$ cells induce ferroptosis in tumor cells, which is the direct evidence of the connection between ferroptosis and antitumor immunity (133). They found that interferon gamma (IFN $\gamma$ ) released from $\mathrm{CD}^{+} \mathrm{T}$ cells downregulated the expression of SLC3A2 and SLC7A11, impaired the uptake of cystine by tumour cells, and promoted ferroptosis. Thus, T cell-promoted tumour ferroptosis is an antitumour mechanism, and targeting this pathway in combination with checkpoint blockade is a potential therapeutic approach.

Furthermore, studies also demonstrated that activating ferroptosis and apoptosis immensely increased chemotherapy sensitivity, which might provide strategies for the combination therapy for cancers. For example, Ye et al. found that the synergy of apoptotic activator and ferroptosis inducer could significantly enhance the cytotoxic effect of gemcitabine in pancreatic cancer, providing a new strategy for pancreatic cancer treatment (134); Hong et al. unveiled a novel treatment strategy for ovarian cancer through a combined use of Poly (ADP-ribose) polymerase S (PARP) inhibitor and ferroptosis inducer (135); Sun et al. identified that QSOX1 (Quiescin sulfhydryl oxidase 1) enhanced sorafenib-induced ferroptosis by promoting the ubiquitination degradation of EGFR and inhibiting EGFR activation and thus inhibiting NRF2, providing QSOX1 as a new candidate target for a sorafenib-based combination therapy in advanced hepatocellular carcinoma or EGFR-dependent tumor types (136). 
There are also some new discoveries focusing on the relationship between lipid peroxidation and ferroptosis. Tan et al. found a hypoxia-inducible factor-dependent adipokine chemerin, which could prevent fatty acid oxidation and lead to escape from ferroptosis, and targeting chemerin reduced lipid storage and tumor growth (137); Lang et al. demonstrated that supplementing unsaturated fatty acids while inhibiting the biogenesis of lipid droplets could induce ferroptosis in acidic cancer cells (138); Additionally, Dierge et al. found that a diet rich in n-3 long-chain unsaturated fatty acids significantly inhibited tumor growth in mice, indicating that supplementation of dietary unsaturated fatty acids can serve as a selective adjuvant anti-tumor approach (139); Furthermore, supplementation of dietary unsaturated fatty acids may serve as a selective adjuvant anti-tumor approach. W. Liu et al. verified that dyslipidemia affected the occurrence and development of tumors by selectively resisting ferroptosis, which primarily due to the continued expression of GPX4 in the metabolism of 27hydroxycholesterol-resistant cells (140); In consistent, Beatty et al. identified conjugated linoleic acids, including $\alpha$ eleostearic acid ( $\alpha \mathrm{ESA}$ ), acted as inducers of ferroptosisby acyl coenzyme a synthetase long-chain isomer 1 and interfering with the biosynthesis of triglycerides inhibited $\alpha E S A$-induced ferroptosis, but not GPX4-inhibited ferroptosis (141).

Notably, many extracts from plants and herbs also exhibit anti-tumor effects by inducing ferroptosis. Z.X.Wang et al. found that quercetin could promote the degradation of lysosomaldependent ferritin and the release of free iron, this effect and quercetin-induced ROS production synergistically led to lipid peroxidation and ferroptosis (142). C.Y. Wu et al. found that Dihydroisotanshinone I (Radix Salviae Miltiorrhizae extract) could induce ferroptosis in lung cancer cells by blocking the expression of GPX4 protein (143). And Wen et al. confirmed that 18 - $\beta$-glycyrrhetinic acid could promote the production of ROS and RNS by activating NADPH oxidase and iNOS, and reducing GSH and GPX activities in triple-negative breast cancer cells, thereby accelerating lipid peroxidation and leading to ferroptosis (144).

\section{Iron and Ferritinophagy}

Ferritinophagy is a type of cell selective autophagy, in which the ferritin (mainly ferritin heavy chain 1) is degraded in autophagosomes mediated by nuclear receptor coactivator 4 (NCOA4), leading to the ferritin-bound iron to be released as free iron $(145,146)$ (Figure 4). Ferritinophagy contributes to the initiation of ferroptosis through degradation of ferritin, which triggers labile iron overload (IO), lipid peroxidation, membrane damage, and cell death (147), and plays a certain role in tumorigenesis.

Ferritin is a complex that can hold $4,500 \mathrm{Fe}^{3+}$ and is widely present in mammalian cells. The ferritin complex is assembled by the heavy chain (ferritin heavy chain 1, FTH1) and the light chain (ferritin light chain, FTL) (148). FTH1 has a ferrous oxidase activity and can catalyze the oxidation of $\mathrm{Fe}^{2+}$ to $\mathrm{Fe}^{3+}$, thereby reducing a large number of $\mathrm{Fe}^{2+}$-produced free radicals participating in the Fenton reaction, and the damage of free radicals to tissues and organs $(149,150)$. Iron combines with

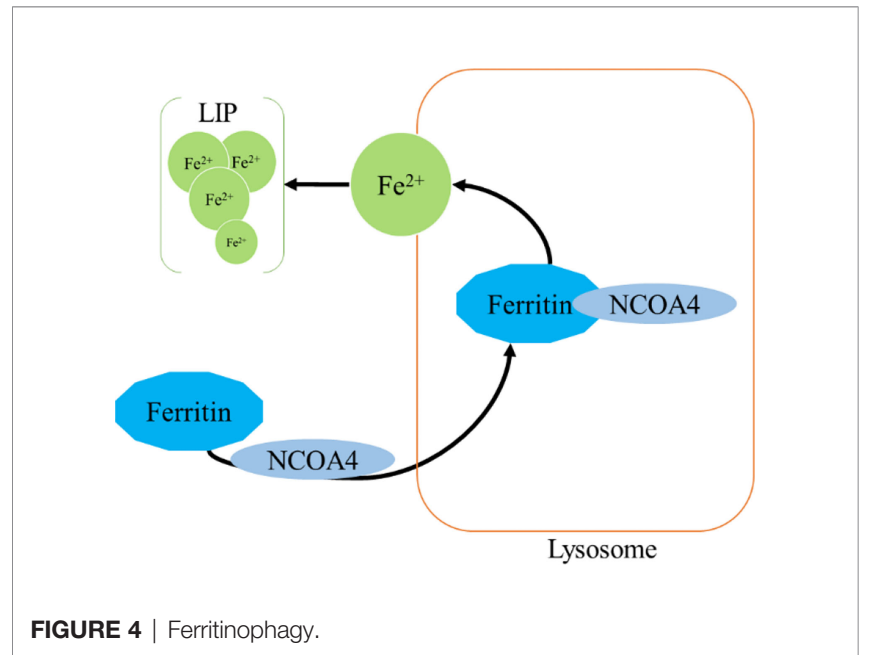

each other through ferritin iron pores and $\mathrm{Fe}^{2+}$ is further oxidized to $\mathrm{Fe}^{3+}$ by FTH1 in the ferritin cage, resulting in the inert deposition of $\mathrm{Fe}^{3+}$, which cannot use or generate ROS in cells (151).The main way of ferritin to release iron is selective autophagy mediated by NCOA4, which binds to ferritin and is transported to lysosomes, where ferritin is degraded and iron is released for cell use (145). NCOA4 selectively interacts with FTH1 subunit of ferritin through its conserved C-terminal domain and key residues on FTH1 (152). Notably, NCOA4 is regulated by HECT and the RLD domain containing E3 ubiquitin protein ligase2 (HERC2) (153).

Mechanistically, when cellular iron level is high, NCOA4 and HERC2 have an iron-dependent effect, resulting in the degradation of NCOA4 through ubiquitin proteasome system, thereby reducing the release of iron and facilitating the storage of ferritin. On the contrary, under low cellular iron conditions, the interaction between NCOA4 and HERC2 is reduced, leading to an increase of NCOA4 level, which increases the degradation of ferritin and iron autophagy flux to supplement cellular iron (154, 155). Thus, a proper amount of ferritinophagy can maintain the balance of iron in cells. However, an excessive activation of ferritinophagy can lead to disease induced by iron overload. Importantly, studies have found that there is a close link between iron release caused by ferritinophagy and ROS damage in ferroptosis $(115,156,157)$. Under certain conditions, iron release caused by ferritinophagy is a component of ferroptosis, and may be a direct driving factor of ferroptosis $(115,156,157)$. The role of ferritinophagy-related genes in cancer progression has been confirmed (158-164). Here, we focus on clarifying the new discovers of NCOA4 and FTH1 in cancer.

The expression of NCOA4 is lower in Clear cell renal cell carcinoma (ccRCC) tissues compared with normal tissues, and low NCOA4 expression is closely related to high-grade malignant tumors and advanced TNM staging (165, 166). Some studies have indicated the critical role of NCOA4mediated ferritinophagy in tumor progression, such as knockdown of COPZ1 leads to an increase in NCOA4, resulting in the degradation of ferritin, and ultimately ferroptosis in glioblastoma (167); Vara-Pérez et al. found that 
melanoma cells lacking BNIP3 showed increased intracellular iron levels caused by NCOA4-mediated increase in ferritinophagy (168). Nevertheless, NCOA4-mediated ferritinophagy is not effective in all tumors as Hasan et al. found that the destruction of ferritinophagy by NCOA4 knockout resulted in minor differences in growth under basal and iron-restricted conditions in colon cancer cells; Additionally, NCOA4 does not engage in cell death induced by 5 -fluorouracil and erastin (169).

Furthermore, FTH1 is also another important gene involved in ferritinophagy. Hayashima et al. demonstrated that in glioblastoma cells, cystine deprivation rather than L-buthionine sulfoximine treatment caused ferroptosis, although they both lead to depletion of cellular GSH, and NCOA4-mediated degradation of FTH1 was observed in cystine-deprived cells but not in L-buthionine sulfoximine -treated cells. Besides, inhibition of FTH1 degradation suppressed cystine deprivation-induced ferroptosis. This suggests that cystine deprivation-induced ferroptosis required not only GSH depletion, but also intracellular iron accumulation, and ferritinophagy plays an essential role in cystine deprivationinduced ferroptosis (170). Notably, due to insufficient FTH1, Erastin or RSL3 induces ferroptosis in neuroblastoma N2A cells, but fails to induce normal nerve cell death, indicating that ferroptosis may be a promising therapeutic target for neuroblastoma (171).

\section{IRON IN CANCER THERAPEUTICS}

\section{Iron in Cancer Prognosis}

Because iron is essential at all stages of tumor development, survival, proliferation, and metastasis, we analyzed the prognostic value of iron and iron regulatory genes. It has shown that serum ferritin has prognostic value in various cancers, and elevated serum FT levels are associated with poor prognosis $(46,172-175)$. However, the increase of serum ferritin are not necessarily associated with upregulated iron level in the body, this means that iron level is regulated by other stimuli, such as inflammation (2). Therefore, we hereby summarize the association between factors related to iron metabolism and prognosis (Table 2).

In view of the important role of TFR1 in iron uptake, TFR1 has been identified as a prognostic marker for many tumors, such as TFR1 expression in $\mathrm{ER}^{-}$tissue was significantly higher than that in $\mathrm{ER}^{+}$tissue (188); Compared with adjacent non-cancer tissues, the mRNA expression level of TFR1 in hepatocellular carcinoma tissues is remarkably increased, and the expression of TFR 1 and TFR2 is negatively correlated with tumor differentiation (176, 177); Furthermore, patients with a high TFR1 expression have a high risk of recurrence and death after hepatectomy (177); Similarly, TFR1 is significantly overexpressed in ovarian cancer and glioblastoma, and TFR1 expression may be related to tumor staging, progression or the short survival time $(29,180)$. Additionally, the activation of EGFR induces cell redistribution of TFR1, and the highly expressed TFR1 is closely related to the progression of lung cancer (178). The assessment of 178 gastric cancer tissues revealed a negative correlation between TFR1 expression and patient prognosis, and the TFR1 positive sorting cells showed a strong proliferative capacity; however, the TFR1negative cells showed a more aggressive tumor features (179). In contrast, although TFR2 is also significantly up-regulated in glioblastoma, but its up-regulation predicts a good prognosis for glioblastoma (181). Notably, in colorectal cancer, patients with a low expression of TFR1 have a shorter survival compared with patients with positive TFR1 expression (182). These results suggest that the roles of TFR $1 / 2$ in tumor prognosis might be tumor-specific.

TABLE 2 | Iron regulators in cancer prognosis.

\begin{tabular}{|c|c|c|c|c|}
\hline Altered Player & Cancer & Regulation & prognosis & Ref. \\
\hline TFR1 & hepatic carcinoma & up & poor & $(176,177)$ \\
\hline TFR1 & ovarian cancer & up & poor & (29) \\
\hline TFR1 & lung cancer & up & poor & $(178)$ \\
\hline TFR1 & gastric cancer & up & poor & $(179)$ \\
\hline TFR1 & glioblastoma & up & poor & $(180)$ \\
\hline TFR2 & glioblastoma & up & good & $(181)$ \\
\hline TFR1 & colorectal cancer & up & good & (182) \\
\hline FPN1 & breast cancer & down & poor & $(45)$ \\
\hline FPN1 & lung cancer & down & poor & $(178)$ \\
\hline FPN1 & adrenocortical carcinoma & down & poor & (183) \\
\hline FPN1 & multiple myeloma & down & poor & $(43)$ \\
\hline FPN1 & clear cell renal cell carcinoma & down & poor & $(184)$ \\
\hline STEAP3 & glioblastoma & up & poor & (39) \\
\hline STEAP1 & breast cancer & up & good & $(185)$ \\
\hline STEAP2 & breast cancer & up & good & (185) \\
\hline STEAP4 & breast cancer & up & good & (185) \\
\hline Hepcidin & breast cancer & up & poor & (186) \\
\hline FTL & ovarian cancer & up & poor & $(187)$ \\
\hline DMT1 & ovarian cancer & up & poor & $(187)$ \\
\hline HAMP & ovarian cancer & up & poor & (187) \\
\hline DMT1 & hepatocellular carcinoma & low & poor & $(65)$ \\
\hline
\end{tabular}


Similarly, FPN1 has been identified as a favorable prognostic marker for many tumors (189). In a gene expression profile of approximately 800 breast cancer patients, it was reported that decreased FPN1 expression was significantly associated with reduced metastasis-free and disease-free survival (45). And FPN1 mRNA and protein expression are significantly downregulated, and patients with a low FPN1 expression have a poor prognosis in lung cancer and adrenocortical carcinoma (ACC) $(178,183)$. Additionally, a similar effect was observed in multiple myeloma and ccRCC $(43,184)$. Furthermore, STEAP3 is highly expressed in glioblastoma and is closely related to the decreased overall survival rate (39); however, a survival analysis showed that breast cancer patients with high levels of STEAP1, STEAP2, and STEAP4 had an overall good prognosis (185).

Higher hepcidin is also reported to be associated with a shorter recurrence time of distant breast cancer, and hepcidin may be associated with a poor prognosis for breast cancer in obese women (186). Survival analysis showed that ovarian cancer patients with a high expression of FTL, DMT1 and HAMP showed a poor overall survival rate (190). IL-6 and BMP control hepcidin secretion in cancer and IL-6 level is elevated in lung cancer patients with poor prognosis (187).

The progression of breast cancer is negatively correlated with hemoglobin $(\mathrm{Hb})$ and positively correlated with ferritin levels (191). Compared with the high-DMT1 group, liver cancer patients with a low DMT1 expression have a worse disease-free survival rate, which is particularly obvious in patients with advanced liver cancer (65).

\section{Iron in Cancer Therapy}

Many approaches have been constructed to treat cancer against intracellular iron metabolism disorders: one strategy is to deplete iron of tumor cells, such as iron chelator; another important one is to generate cytotoxic level of ROS or ferroptosis through excess iron in tumor cells (Table 3 ).

\section{Iron Depletion}

Iron chelators can change the metabolism of tumor cells by reducing the intake of iron. It has also been demonstrated that iron chelators inhibit ribonucleotide reductase activity and play important roles in various signaling pathways associated with tumor progression and metastasis $(212,213)$. In a study of patients with advanced hepatocellular carcinoma, 20\% of patients responded to treatment with DFO (194); In another study of nine patients with neuroblastoma, seven patients had reduced bone marrow infiltration after DFO treatment (195). However, iron chelators appear to be effective only in specific tumors. Other studies have shown that DFO is ineffective in the treatment of hormone-reducing refractory prostate cancer and recurrent neuroblastoma $(214,215)$. Notably, other chelating agents, such as Triapine and Dp44mT, have entered clinical experiments $(216,217)$. After receiving Triapine treatment, $76 \%$ of patients with advanced hematological malignancies had a 50\% reduction in white blood cell count (196). However, Triapine only showed the smallest overall response rate in metastatic renal cell carcinoma and recurrent and metastatic head and neck squamous cell carcinoma $(218,219)$. Additionally, Hui et al.
TABLE 3 | Iron in Cancer Therapy.

\begin{tabular}{llc}
\hline Name & \multicolumn{1}{c}{ Mechanism } & Ref. \\
\hline Cyst(e)inase & degrading cysteine & $(133,192,193)$ \\
DFO & iron depletion & $(194)$ \\
DFO & iron depletion & $(195)$ \\
Triapine & iron depletion & $(196)$ \\
Dp44mT & iron depletion \\
Fusaricide & iron depletion & \\
Ga & iron depletion & $(62)$ \\
Tris (8-quinolinolato) Gallium (III) (KP46) & iron depletion & $(197)$ \\
Gallium Mallotate & iron depletion & $(198)$ \\
Ferrocene derivatives & elevated iron levels & $(198)$ \\
Ferumoxytol & elevated iron levels & $(199)$ \\
ascorbic acid & ferroptosis & $(200)$ \\
salinomycin derivatives & ferroptosis inducers & $(201-205)$ \\
DMT1 inhibitor & target DMT1 & $(68)$ \\
HFn-PTX & target TFR1 & $(207)$ \\
DOX-FTH & target TFR1 & $(179,208)$ \\
miR-375 & target SLC7A11 & $(209)$ \\
miR-148a & targeting TFR1 & $(32)$ \\
miR-184 & targeting HAMP & $(210)$ \\
miR-200b & targeting ferritin & $(211)$ \\
\hline
\end{tabular}

confirmed that Fusaricide, a novel iron chelating agent, could induce apoptosis by activating Caspase-3 (62). Furthermore, iron chelators have been shown to alter macrophage polarization, and immune signaling. Prill et al. showed that DFO administration led to high iron efflux by decreasing ferritin expression in the tumor-associated macrophages (220). EC1 (a thiosemicarbazone chelator) treatment reversed the positive effect of macrophageconditioned media on the proliferation and migration of cancer cells (221). Moreover, the safety and efficacy of iron chelators as cancer therapeutics have been tested in clinical trials, gastrointestinal symptoms and fatigue appear to be the most prevalent toxicities $(194,219,222,223)$.

The use of iron chelators in combination therapy has also been partially investigated. DFO was combined with many different chemotherapeutic drugs, such as cyclophosphamide, etoposide, cisplatin, carboplatin, and thiotepa (190, 224, 225). In a cohort of patients with advanced neuroblastoma and primitive neuroectodermal tumors, DFO was effective in combination with cyclophosphamide, etoposide, carboplatin, and thiotepa (224). In another study of 37 patients with accelerated myeloproliferative tumors and secondary acute myeloid leukemia, the combination of Triapine and fludarabine (a DNA synthesis inhibitor) showed $49 \%$ overall response rate and $24 \%$ complete response rate (226).

Gallium salts, which belong to the group IIIa metals and have common chemical properties with iron, are variants of the iron depletion strategy in tumor therapy. Therefore, gallium $(\mathrm{Ga})$ is used to disrupt iron metabolism to mimic the behavior of iron. Gallium can be incorporated into protein and enzymes that use iron as a cofactor, such as ribonucleotide reductase, which inactivates enzymes that require iron to function and lead to an increase in mitochondrial reactive oxygen species (197). Thus, the gallium-based compound exhibits antitumor activity by disrupting iron-dependent tumor metabolism. The spectrum antineoplastic activity of gallium nitrate in the clinic was evaluated in Phase II clinical trials. Among a number of cancers examined, gallium nitrate displayed promising 
antineoplastic activity in patients with nonHodgkin's lymphoma and bladder cancer (198, 227-229). The anti-cancer activity of newer gallium-ligands have been developed and are being undergone clinical evaluation, such as Tris (8-quinolinolato) Gallium (III) (KP46) and Gallium Mallotate, which may be more effective than the nitrate salt used in the original clinical formulation of gallium $(198,230)$.

\section{Elevated Iron Levels}

The strategy in contrast to iron depletion is to supply cells with excess iron. Excess iron combines with high levels of unstable iron in tumor cells to produce large amounts of ROS to eliminate tumor cells. For example, the metal-containing drugs Ferrocene derivatives are stable and exhibit favorable redox properties, inhibiting proliferative activity of tumor cell lines (199). Additionally, Ferumoxytol is an iron oxide nanoparticle approved by the FDA for the treatment of clinical iron deficiency, and studies have shown that ferumoxytol can produce excessive amounts of free iron, the reactive oxygen species produced by which can cause cell death, increase oxidative stress, and reduce tumor burden cells in mouse leukemia models and patients (200).

What's more, ascorbic acid therapy is a variant of cancer treatment strategy by affecting the oxidation state of iron and increasing LIP levels, which is indicated for various tumors (201203). Multiple clinical trials of this therapy are currently being pursued. Such as NCT02344355, NCT02420314, NCT02905578, NCT02905591, NCT03508726, NCT03602235, and NCT03799094 (https://clinicaltrials.gov/) (204, 205). However, it must be noted that if a large amount of divalent iron is oxidized to trivalent iron, you will suffer from methemoglobinemia. Furthermore, high levels of iron in the blood reduce our healthy years of life, and keeping these levels in check could prevent age-related damage (231).

\section{Ferroptosis Inducers}

The ferroptosis inducers can target the treatment of tumor by increasing the iron level of the tumor cells. Conversely, targeting ferroptosis inhibitors also have been shown to suppress tumor progression, for example, depletion of cystine or cystine by cyst (e)inase (an engineered enzyme that degrades both cystine and cysteine) in combination with checkpoint blockade synergistically enhances $\mathrm{T}$ cell-mediated anti-tumour immunity and induces ferroptosis in tumor cells (133); Cyst(e)inase also can synergize with thioredoxin reductase inhibition for suppression of pancreatic tumor growth (192); Additionally, administration of cyst(e)inase induces tumor-selective ferroptosis and inhibited pancreatic tumor growth (193). IFN- $\gamma$ derived from immunotherapy-activated $\mathrm{CD}^{+} \mathrm{T}$ cells synergizing with radiotherapy-activated ataxia-telangiectasia mutated (ATM) suppresses SLC7A11, to induce cystine uptake, enhance tumor lipid oxidation and ferroptosis in human fibrosarcoma and melanoma cells (232). Notably, as we and others previously showed that that ferroptosis inducers target tumor stem cells to inhibit tumor proliferation and reduce metastasis $(205,233,234)$. Several US Food and Drug Administration (FDA)-approved drugs have been shown to induce ferroptosis in tumor cells in preclinical models, but the clinical utility of these ferroptosis inducers require further investigation (2). Another study has shown that salinomycin derivatives can trigger the ferroptosis pathway in cancer stem cells (206). Furthermore, Andreea L Turcu et al. developed a DMT1 inhibitor that selectively targets cancer stem cells by blocking lysosomal iron translocation, leading to lysosomal iron accumulation, ROS production, and ferroptosis (68). However, it must be noted that ferroptosis inducers have potentially harmful side effects, mainly manifested as DNA damage and cell death in normal bone marrow cells and various tissues $(235,236)$.

In addition to the above two strategies, another strategy is to directly target proteins related to iron homeostasis disorders. Such as methods that target TFR1, which is commonly overexpressed in tumor cell species. W. Liu et al. developed endogenous human ferritin heavy-chain nanocages (HFn) to serve as the carrier of paclitaxel (PTX), which can specifically bind to blood-brain barrier and TFR1 widely overexpressed in glioma cells, and showed that HFn-PTX showed the best antitumor effect, and the median survival time was significantly longer than that of free PTX (207); additionally, doxorubicinloaded ferritin heavy chain (DOX-FTH) can be taken up and induce apoptosis of cancer cells overexpressing TFR1 (208); and Cheng et al. indicated that HFn-Dox treatment could significantly improve the therapeutic effect of doxorubicin on gastric cancer, and increase overall survival rate of tumorbearing mice (179).

Another possible new treatment strategy is to target miRNAs that regulate iron metabolism or to use miRNA in combination with chemotherapeutic drugs. Such as, our laboratory observed that miR-375 can trigger the ferroptosis through targeting SLC7A11, which is essential for miR-375-mediated inhibition on gastric cancer cell stemness (209); Overexpression of miR148 a can inhibit the proliferation of liver cancer cells by targeting TFR1 (32); MiR-184 can inhibit the occurrence and development of liver cancer by regulating the expression of HAMP (210); And overexpression of miR-200b can reduce ferritin levels in vitro and increase the sensitivity of cancer cells to doxorubicin (211).

\section{PERSPECTIVES AND FUTURE DIRECTIONS}

Iron is necessary for normal cell metabolism, while as a redox active metal, iron can also produce active oxygen, which is a potentially toxic substance. In recent years, there has been an exPLoSive growth in the research of iron homeostasis in normal cells and iron metabolism disorders in cancer. In most tumors, cancer cells retain iron metabolism pathways similar to those of normal cells, but the levels of many proteins and enzyme activities are changed, which indicates that reprogramming of iron metabolism is an important aspect of tumor cell survival. The increase of iron levels in cancer cells promotes the activity of iron-dependent proteins, while avoids the damage caused by iron overload and achieves the "adjusted iron homeostasis" in line with tumor metabolism. However, the specific mechanism is not yet fully understood, such as is the iron metabolism of different types of tumor cells 
consistent? How to quantify the level of iron so as to specifically target tumor cells without harming normal cells?

It may be helpful to solve these problems by developing powerful quantitative methods to measure the metabolizable iron in the cytoplasm and organelles, as well as developing iron chelating agents for organelles. Additionally, currently, the main treatment is to trigger the apoptotic death of tumor cells with anticancer drugs. However, due to the intrinsic and acquired resistance of tumor cells to apoptosis, the therapeutic effect is limited, and drug resistance is still the main limiting factor for the cure of tumor patients. Therefore, using other forms of non-apoptotic cell death provides a new therapeutic strategy. Importantly, abnormal lipid metabolism, ROS accumulation, and iron addiction are the physiological differences between malignant tumor cells and normal cells, and these differences happen to be the key regulatory factors of ferroptosis. Therefore, compared with normal cells, tumor cells are more sensitive to ferroptosis. From our own perspectives, ferroptosis inducers could have a worthwhile therapeutic index. However, based on different metabolic states and expression levels of key regulatory proteins, different tumor cells might have different sensitivity and response to ferroptosis. Notably, increasing evidences showed that combined use of ferroptosis inducer and other treatments, such as chemotherapy, radiotherapy, and immunotherapy, is a good treatment strategy, especially for cancer stem cells and drug-resistant cells. Thus, it is expected that cancer cell iron addiction will be successfully used as an effective cancer treatment in the next few years, such as: introducing drugs that induce ferroptosis into the clinic, transforming the unique iron metabolism characteristics of cancer stem cells into therapeutic advantages, and developing more targeted and effective anti-cancer iron chelating agents.

\section{AUTHOR CONTRIBUTIONS}

QG, SH, and ZY reviewed the literature and drafted the article. LL, XL, CL, WZ, and LZ finalized the paper and provided suggestions to improve it. All authors participated in designing the concept of this manuscript. All authors contributed to the article and approved the submitted version.

\section{FUNDING}

This work was supported by the National Natural Science Foundation of China (no. 81903857), the Science and Technology Research Project of Henan Province (no. 202102310158); the Medical Science and Technology Research Project of Henan Province (no. LHGJ20190675); the Medical Science and Technology Research Project of Henan Province (no. SBGJ202003010); the 2019 Ningbo Natural Science Foundation (2019A610311); the Doctoral Research Start-up Foundation of Henan Cancer Hospital, and the Priority Academic Program Development (PAPD) of Jiangsu Higher Education Institutions.

\section{REFERENCES}

1. Vogt AS, Arsiwala T, Mohsen M, Vogel M, Manolova V, Bachmann MF. On Iron Metabolism and Its Regulation. Int J Mol Sci (2021) 22(9):4591. doi: 10.3390/ijms22094591

2. Torti SV, Manz DH, Paul BT, Blanchette-Farra N, Torti FM. Iron and Cancer. Annu Rev Nutr (2018) 38:97-125. doi: 10.1146/annurev-nutr-082117-051732

3. Torti SV, Torti FM. Iron and Cancer: More Ore to be Mined. Nat Rev Cancer (2013) 13(5):342-55. doi: 10.1038/nrc3495

4. Jung M, Mertens C, Tomat E, Brüne B. Iron as a Central Player and Promising Target in Cancer Progression. Int J Mol Sci (2019) 20(2):273. doi: 10.3390/ijms20020273

5. Seco-Cervera M, González-Cabo P, Pallardó FV, Romá-Mateo C, GarcíaGiménez JL. Thioredoxin and Glutaredoxin Systems as Potential Targets for the Development of New Treatments in Friedreich's Ataxia. Antioxid (Basel Switzerland) (2020) 9(12):1257. doi: 10.3390/antiox9121257

6. Tifoun N, De Las Heras JM, Guillaume A, Bouleau S, Mignotte B, Le Floch N. Insights Into the Roles of the Sideroflexins/SLC56 Family in Iron Homeostasis and Iron-Sulfur Biogenesis. Biomedicines (2021) 9(2):103. doi: 10.3390/biomedicines9020103

7. Jomova K, Valko M. Advances in Metal-Induced Oxidative Stress and Human Disease. Toxicology (2011) 283(2-3):65-87. doi: 10.1016/j.tox.2011.03.001

8. Filipovic MR, Koppenol WH. The Haber-Weiss Reaction - The Latest Revival. Free Radical Biol Med (2019) 145:221-2. doi: 10.1016/ j.freeradbiomed.2019.09.017

9. Lipinski B. Hydroxyl Radical and Its Scavengers in Health and Disease. Oxid Med Cell Longevity (2011) 2011:809696. doi: 10.1155/2011/809696

10. Reiter RJ, Mayo JC, Tan DX, Sainz RM, Alatorre-Jimenez M, Qin L. Melatonin as an Antioxidant: Under Promises But Over Delivers. J Pineal Res (2016) 61(3):253-78. doi: 10.1111/jpi.12360

11. Abbaspour N, Hurrell R, Kelishadi R. Review on Iron and Its Importance for Human Health. J Res Med Sci (2014) 19(2):164-74.

12. Kawabata H. Transferrin and Transferrin Receptors Update. Free Radical Biol Med (2019) 133:46-54. doi: 10.1016/j.freeradbiomed.2018. 06.037

13. Bogdan AR, Miyazawa M, Hashimoto K, Tsuji Y. Regulators of Iron Homeostasis: New Players in Metabolism, Cell Death, and Disease. Trends Biochem Sci (2016) 41(3):274-86. doi: 10.1016/j.tibs.2015.11.012

14. Ginzburg YZ. Hepcidin-Ferroportin Axis in Health and Disease. Vitamins Hormones (2019) 110:17-45. doi: 10.1016/bs.vh.2019.01.002

15. Zhang DL, Ghosh MC, Rouault TA. The Physiological Functions of Iron Regulatory Proteins in Iron Homeostasis - An Update. Front Pharmacol (2014) 5:124. doi: 10.3389/fphar.2014.00124

16. Anderson CP, Shen M, Eisenstein RS, Leibold EA. Mammalian Iron Metabolism and Its Control by Iron Regulatory Proteins. Biochim Biophys Acta (2012) 1823(9):1468-83. doi: 10.1016/j.bbamcr.2012.05.010

17. Hentze MW, Muckenthaler MU, Galy B, Camaschella C. Two to Tango: Regulation of Mammalian Iron Metabolism. Cell (2010) 142(1):24-38. doi: 10.1016/j.cell.2010.06.028

18. Petronek MS, Spitz DR, Buettner GR, Allen BG. Linking Cancer Metabolic Dysfunction and Genetic Instability Through the Lens of Iron Metabolism. Cancers (2019) 11(8):1077. doi: 10.3390/cancers11081077

19. Anderson GJ, Frazer DM. Hepatic Iron Metabolism. Semin Liver Dis (2005) 25(4):420-32. doi: 10.1055/s-2005-923314

20. Rishi G, Subramaniam VN. The Liver in Regulation of Iron Homeostasis. Am J Physiol Gastrointest Liver Physiol (2017) 313(3):G157-g65. doi: 10.1152/ ajpgi.00004.2017

21. Qiao B, Sugianto P, Fung E, Del-Castillo-Rueda A, Moran-Jimenez MJ, Ganz T, et al. Hepcidin-Induced Endocytosis of Ferroportin Is Dependent on Ferroportin Ubiquitination. Cell Metab (2012) 15(6):918-24. doi: 10.1016/j.cmet.2012.03.018 
22. Andriopoulos BJr., Corradini E, Xia Y, Faasse SA, Chen S, Grgurevic L, et al. BMP6 Is a Key Endogenous Regulator of Hepcidin Expression and Iron Metabolism. Nat Genet (2009) 41(4):482-7. doi: 10.1038/ng.335

23. Pietrangelo A. Ferroportin Disease: Pathogenesis, Diagnosis and Treatment. Haematologica (2017) 102(12):1972-84. doi: 10.3324/haematol.2017.170720

24. Gao G, Li J, Zhang Y, Chang YZ. Cellular Iron Metabolism and Regulation. Adv Exp Med Biol (2019) 1173:21-32. doi: 10.1007/978-981-13-9589-5_2

25. Prior R, Reifenberger G, Wechsler W. Transferrin Receptor Expression in Tumours of the Human Nervous System: Relation to Tumour Type, Grading and Tumour Growth Fraction. Virchows Archiv A Pathol Anat Histopathol (1990) 416(6):491-6. doi: 10.1007/BF01600299

26. Das Gupta A, Shah VI. Correlation of Transferrin Receptor Expression With Histologic Grade and Immunophenotype in Chronic Lymphocytic Leukemia and Non-Hodgkin's Lymphoma. Hematol Pathol (1990) 4(1):37-41.

27. Faulk WP, Hsi BL, Stevens PJ. Transferrin and Transferrin Receptors in Carcinoma of the Breast. Lancet (Lond Engl) (1980) 2(8191):390-2. doi: 10.1016/S0140-6736(80)90440-7

28. Zhang S, Chang W, Wu H, Wang YH, Gong YW, Zhao YL, et al. Pan-Cancer Analysis of Iron Metabolic Landscape Across the Cancer Genome Atlas. J Cell Physiol (2020) 235(2):1013-24. doi: 10.1002/jcp.29017

29. Basuli D, Tesfay L, Deng Z, Paul B, Yamamoto Y, Ning G, et al. Iron Addiction: A Novel Therapeutic Target in Ovarian Cancer. Oncogene (2017) 36(29):4089-99. doi: 10.1038/onc.2017.11

30. Wang Q, Gu T, Ma L, Bu S, Zhou W, Mao G, et al. Efficient Iron Utilization Compensates for Loss of Extracellular Matrix of Ovarian Cancer Spheroids. Free Radical Biol Med (2021) 164:369-80. doi: 10.1016/j.freeradbiomed.2021.01.001

31. Xiao C, Fu X, Wang Y, Liu H, Jiang Y, Zhao Z, et al. Transferrin Receptor Regulates Malignancies and the Stemness of Hepatocellular CarcinomaDerived Cancer Stem-Like Cells by Affecting Iron Accumulation. PLoS One (2020) 15(12):e0243812. doi: 10.1371/journal.pone.0243812

32. Babu KR, Muckenthaler MU. miR-148a Regulates Expression of the Transferrin Receptor 1 in Hepatocellular Carcinoma. Sci Rep (2019) 9 (1):1518. doi: 10.1038/s41598-018-35947-7

33. Campisi A, Bonfanti R, Raciti G, Bonaventura G, Legnani L, Magro G, et al. Gene Silencing of Transferrin-1 Receptor as a Potential Therapeutic Target for Human Follicular and Anaplastic Thyroid Cancer. Mol Ther Oncol (2020) 16:197-206. doi: 10.1016/j.omto.2020.01.003

34. Fu Y, Lin L, Xia L. MiR-107 Function as a Tumor Suppressor Gene in Colorectal Cancer by Targeting Transferrin Receptor 1. Cell Mol Biol Lett (2019) 24:31. doi: 10.1186/s11658-019-0155-Z

35. Xue X, Ramakrishnan SK, Weisz K, Triner D, Xie L, Attili D, et al. Iron Uptake via DMT1 Integrates Cell Cycle With JAK-STAT3 Signaling to Promote Colorectal Tumorigenesis. Cell Metab (2016) 24(3):447-61. doi: 10.1016/j.cmet.2016.07.015

36. Sideris M, Adams K, Moorhead J, Diaz-Cano S, Bjarnason I, Papagrigoriadis S. BRAF V600E Mutation in Colorectal Cancer Is Associated With RightSided Tumours and Iron Deficiency Anaemia. Anticancer Res (2015) 35 (4):2345-50.

37. Liu Y, Pang Y, Zhu B, Uher O, Caisova V, Huynh TT, et al. Therapeutic Targeting of SDHB-Mutated Pheochromocytoma/Paraganglioma With Pharmacologic Ascorbic Acid. Clin Cancer Res (2020) 26(14):3868-80. doi: 10.1158/1078-0432.CCR-19-2335

38. Xue X, Taylor M, Anderson E, Hao C, Qu A, Greenson JK, et al. HypoxiaInducible Factor-2 $\alpha$ Activation Promotes Colorectal Cancer Progression by Dysregulating Iron Homeostasis. Cancer Res (2012) 72(9):2285-93. doi: 10.1158/0008-5472.CAN-11-3836

39. Han M, Xu R, Wang S, Yang N, Ni S, Zhang Q, et al. Six-Transmembrane Epithelial Antigen of Prostate 3 Predicts Poor Prognosis and Promotes Glioblastoma Growth and Invasion. Neoplasia (New York NY) (2018) 20 (6):543-54. doi: 10.1016/j.neo.2018.04.002

40. Zhang L, Ye Y, Tu H, Hildebrandt MA, Zhao L, Heymach JV, et al. MicroRNA-Related Genetic Variants in Iron Regulatory Genes, Dietary Iron Intake, microRNAs and Lung Cancer Risk. Ann Oncol (2017) 28 (5):1124-9. doi: 10.1093/annonc/mdx046

41. Fehringer G, Liu G, Pintilie M, Sykes J, Cheng D, Liu N, et al. Association of the 15 q25 and 5p15 Lung Cancer Susceptibility Regions With Gene Expression in Lung Tumor Tissue. Cancer Epidemiol Biomarkers Prev (2012) 21(7):1097-104. doi: 10.1158/1055-9965.EPI-11-1123-T
42. Deng Z, Manz DH, Torti SV, Torti FM. Effects of Ferroportin-Mediated Iron Depletion in Cells Representative of Different Histological Subtypes of Prostate Cancer. Antioxid Redox Signaling (2019) 30(8):1043-61. doi: 10.1089/ars.2017.7023

43. Kong Y, Hu L, Lu K, Wang Y, Xie Y, Gao L, et al. Ferroportin Downregulation Promotes Cell Proliferation by Modulating the Nrf2miR-17-5p Axis in Multiple Myeloma. Cell Death Dis (2019) 10(9):624. doi: 10.1038/s41419-019-1854-0

44. Zhou Q, Chen J, Feng J, Wang J. E4BP4 Promotes Thyroid Cancer Proliferation by Modulating Iron Homeostasis Through Repression of Hepcidin. Cell Death Dis (2018) 9(10):987. doi: 10.1038/s41419-018-1001-3

45. Pinnix ZK, Miller LD, Wang W, D'Agostino RJr., Kute T, Willingham MC, et al. Ferroportin and Iron Regulation in Breast Cancer Progression and Prognosis. Sci Trans Med (2010) 2(43):43ra56. doi: 10.1126/scitranslmed. 3001127

46. Sornjai W, Nguyen Van Long F, Pion N, Pasquer A, Saurin JC, Marcel V, et al. Iron and Hepcidin Mediate Human Colorectal Cancer Cell Growth. Chemico-Biol Interact (2020) 319:109021. doi: 10.1016/j.cbi.2020.109021

47. Tesfay L, Clausen KA, Kim JW, Hegde P, Wang X, Miller LD, et al. Hepcidin Regulation in Prostate and Its Disruption in Prostate Cancer. Cancer Res (2015) 75(11):2254-63. doi: 10.1158/0008-5472.CAN-14-2465

48. Zhao B, Li R, Cheng G, Li Z, Zhang Z, Li J, et al. Role of Hepcidin and Iron Metabolism in the Onset of Prostate Cancer. Oncol Lett (2018) 15(6):9953-8. doi: 10.3892/ol.2018.8544

49. El-Mahdy RI, Zakhary MM, Maximous DW, Mokhtar AA, El Dosoky MI. Circulating Osteocyte-Related Biomarkers (Vitamin D, Sclerostin, Dickkopf-1), Hepcidin, and Oxidative Stress Markers in Early Breast Cancer: Their Impact in Disease Progression and Outcome. J Steroid Biochem Mol Biol (2020) 204:105773. doi: 10.1016/j.jsbmb.2020.105773

50. Lopes M, Duarte TL, Teles MJ, Mosteo L, Chacim S, Aguiar E, et al. Loss of Erythroblasts in Acute Myeloid Leukemia Causes Iron Redistribution With Clinical Implications. Blood Adv (2021) 5(16):3102-12. doi: 10.1182/ bloodadvances. 2021004373

51. Maes K, Nemeth E, Roodman GD, Huston A, Esteve F, Freytes C, et al. In Anemia of Multiple Myeloma, Hepcidin Is Induced by Increased Bone Morphogenetic Protein 2. Blood (2010) 116(18):3635-44. doi: 10.1182/ blood-2010-03-274571

52. Hara M, Ando M, Tsuchiya K, Nitta K. Serum Hepcidin-25 Level Linked With High Mortality in Patients With Non-Hodgkin Lymphoma. Ann Hematol (2015) 94(4):603-8. doi: 10.1007/s00277-014-2255-1

53. Traeger L, Ellermann I, Wiethoff H, Ihbe J, Gallitz I, Eveslage M, et al. Serum Hepcidin and GDF-15 Levels as Prognostic Markers in Urothelial Carcinoma of the Upper Urinary Tract and Renal Cell Carcinoma. BMC Cancer (2019) 19(1):74. doi: 10.1186/s12885-019-5278-0

54. Wang J, Liu W, Li JC, Li M, Li B, Zhu R. Hepcidin Downregulation Correlates With Disease Aggressiveness And Immune Infiltration in Liver Cancers. Front Oncol (2021) 11:714756. doi: 10.3389/fonc.2021.714756

55. Shen Y, Li X, Su Y, Badshah SA, Zhang B, Xue Y, et al. HAMP Downregulation Contributes to Aggressive Hepatocellular Carcinoma via Mechanism Mediated by Cyclin4-Dependent Kinase-1/STAT3 Pathway. Diagn (Basel Switzerland) (2019) 9(2):48. doi: 10.3390/diagnostics 9020048

56. Wang Z, Du Y. Identification of a Novel Mutation Gene Signature HAMP for Cholangiocarcinoma Through Comprehensive TCGA and GEO Data Mining. Int Immunopharmacol (2021) 99:108039. doi: 10.1016/ j.intimp.2021.108039

57. Schieber M, Chandel NS. ROS Function in Redox Signaling and Oxidative Stress. Curr Biol CB (2014) 24(10):R453-62. doi: 10.1016/ j.cub.2014.03.034

58. Matthew-Onabanjo AN, Janusis J, Mercado-Matos J, Carlisle AE, Kim D, Levine F, et al. Beclin 1 Promotes Endosome Recruitment of Hepatocyte Growth Factor Tyrosine Kinase Substrate to Suppress Tumor Proliferation. Cancer Res (2020) 80(2):249-62. doi: 10.1158/0008-5472.CAN-19-1555

59. Bajbouj K, Shafarin J, Hamad M. Estrogen-Dependent Disruption of Intracellular Iron Metabolism Augments the Cytotoxic Effects of Doxorubicin in Select Breast and Ovarian Cancer Cells. Cancer Manage Res (2019) 11:4655-68. doi: 10.2147/CMAR.S204852

60. Wang B, Zhang J, Song F, Tian M, Shi B, Jiang H, et al. EGFR Regulates Iron Homeostasis to Promote Cancer Growth Through Redistribution of 
Transferrin Receptor 1. Cancer Lett (2016) 381(2):331-40. doi: 10.1016/ j.canlet.2016.08.006

61. Jung SY, Lee HK, Kim H, Kim S, Kim JS, Kang JG, et al. Depletion of ST6GALNACIII Retards A549 Non-Small Cell Lung Cancer Cell Proliferation by Downregulating Transferrin Receptor Protein 1 Expression. Biochem Biophys Res Commun (2021) 575:78-84. doi: $10.1016 /$ j.bbrc.2021.08.055

62. Hui Y, Tang T, Wang J, Zhao H, Yang HY, Xi J, et al. Fusaricide Is a Novel Iron Chelator That Induces Apoptosis Through Activating Caspase-3. J Natural Products (2021) 84(8):2094-103. doi: 10.1021/acs.jnatprod. 0c01322

63. Martínez LE, Daniels-Wells TR, Guo Y, Magpantay LI, Candelaria PV, Penichet ML, et al. Targeting TfR1 With the Ch128.1/IgG1 Antibody Inhibits EBV-Driven Lymphomagenesis in Immunosuppressed Mice Bearing EBV(+) Human Primary B-Cells. Mol Cancer Ther (2021) 20 (9):1592-602. doi: 10.1158/1535-7163.MCT-21-0074

64. Yang H, Said AM, Huang H, Papa APD, Jin G, Wu S, et al. Chlorogenic Acid Depresses Cellular Bioenergetics to Suppress Pancreatic Carcinoma Through Modulating C-Myc-TFR1 Axis. Phytother Res PTR (2021) 35 (4):2200-10. doi: 10.1002/ptr.6971

65. Hoki T, Katsuta E, Yan L, Takabe K, Ito F. Low DMT1 Expression Associates With Increased Oxidative Phosphorylation and Early Recurrence in Hepatocellular Carcinoma. J Surg Res (2019) 234:343-52. doi: 10.1016/ j.jss.2018.11.008

66. Chen C, Wang S, Liu P. Deferoxamine Enhanced Mitochondrial Iron Accumulation and Promoted Cell Migration in Triple-Negative MDAMB-231 Breast Cancer Cells Via a ROS-Dependent Mechanism. Int J Mol Sci (2019) 20(19):4952. doi: 10.3390/ijms20194952

67. Chen C, Liu P, Duan X, Cheng M, Xu LX. Deferoxamine-Induced High Expression of TfR1 and DMT1 Enhanced Iron Uptake in Triple-Negative Breast Cancer Cells by Activating IL-6/PI3K/AKT Pathway. OncoTargets Ther (2019) 12:4359-77. doi: 10.2147/OTT.S193507

68. Turcu AL, Versini A, Khene N, Gaillet C, Cañeque T, Müller S, et al. DMT1 Inhibitors Kill Cancer Stem Cells by Blocking Lysosomal Iron Translocation. Chem (Weinheim an der Bergstrasse Germany) (2020) 26(33):7369-73. doi: 10.1002/chem.202000159

69. Yang C, Xia Z, Li T, Chen Y, Zhao M, Sun Y, et al. Antioxidant Effect of Propofol in Gliomas and Its Association With Divalent Metal Transporter 1. Front Oncol (2020) 10:590931. doi: 10.3389/fonc.2020.590931

70. Grunewald TG, Bach H, Cossarizza A, Matsumoto I. The STEAP Protein Family: Versatile Oxidoreductases and Targets for Cancer Immunotherapy With Overlapping and Distinct Cellular Functions. Biol Cell (2012) 104 (11):641-57. doi: 10.1111/boc.201200027

71. Chen H, Xu C, Yu Q, Zhong C, Peng Y, Chen J, et al. Comprehensive Landscape of STEAP Family Functions and Prognostic Prediction Value in Glioblastoma. J Cell Physiol (2021) 236(4):2988-3000. doi: 10.1002/jcp.30060

72. Burnell SEA, Spencer-Harty S, Howarth S, Bodger O, Kynaston H, Morgan C, et al. Utilisation of the STEAP Protein Family in a Diagnostic Setting may Provide a More Comprehensive Prognosis of Prostate Cancer. PLoS One (2019) 14(8):e0220456. doi: 10.1371/journal.pone.0220456

73. Valenti MT, Dalle Carbonare L, Donatelli L, Bertoldo F, Giovanazzi B, Caliari F, et al. STEAP mRNA Detection in Serum of Patients With Solid Tumours. Cancer Lett (2009) 273(1):122-6. doi: 10.1016/j.canlet.2008.07.037

74. Isobe T, Baba E, Arita S, Komoda M, Tamura S, Shirakawa T, et al. Human STEAP3 Maintains Tumor Growth Under Hypoferric Condition. Exp Cell Res (2011) 317(18):2582-91. doi: 10.1016/j.yexcr.2011.07.022

75. Na H, Li X, Zhang X, Xu Y, Sun Y, Cui J, et al. IncRNA STEAP3-AS1 Modulates Cell Cycle Progression via Affecting CDKN1C Expression Through STEAP3 in Colon Cancer. Mol Ther Nucleic Acids (2020) 21:480-91. doi: 10.1016/j.omtn.2020.06.011

76. Wang Z, Guo R, Trudeau SJ, Wolinsky E, Ast T, Liang JH, et al. CYB561A3 Is the Key Lysosomal Iron Reductase Required for Burkitt B-Cell Growth and Survival. Blood (2021) 2021011079. doi: 10.1182/blood.2021011079

77. Burnell SEA, Spencer-Harty S, Howarth S, Bodger O, Kynaston H, Morgan C, et al. STEAP2 Knockdown Reduces the Invasive Potential of Prostate Cancer Cells. Sci Rep (2018) 8(1):6252. doi: 10.1038/s41598-018-24655-x

78. Jiao Q, Du X, Wei J, Li Y, Jiang H. Oxidative Stress Regulated Iron Regulatory Protein IRP2 Through FBXL5-Mediated Ubiquitination-
Proteasome Way in SH-SY5Y Cells. Front Neurosci (2019) 13:20. doi: 10.3389 /fnins.2019.00020

79. Wang H, Shi H, Rajan M, Canarie ER, Hong S, Simoneschi D, et al. FBXL5 Regulates IRP2 Stability in Iron Homeostasis via an Oxygen-Responsive [2fe2s] Cluster. Mol Cell (2020) 78(1):31-41.e5. doi: 10.1016/j.molcel.2020.02.011

80. Song J, Liu T, Yin Y, Zhao W, Lin Z, Yin Y, et al. The Deubiquitinase OTUD1 Enhances Iron Transport and Potentiates Host Antitumor Immunity. EMBO Rep (2021) 22(2):e51162. doi: 10.15252/embr.202051162

81. Zhang Y, Feng X, Zhang J, Chen X. Iron Regulatory Protein 2 Exerts Its Oncogenic Activities by Suppressing TAp63 Expression. Mol Cancer Res MCR (2020) 18(7):1039-49. doi: 10.1158/1541-7786.MCR-19-1104

82. Zhang J, Kong X, Zhang Y, Sun W, Xu E, Chen X. Mdm2 is a Target and Mediator of IRP2 in Cell Growth Control. FASEB J (2020) 34(2):2301-11. doi: 10.1096/fj.201902278RR

83. Horniblow RD, Bedford M, Hollingworth R, Evans S, Sutton E, Lal N, et al. BRAF Mutations Are Associated With Increased Iron Regulatory Protein-2 Expression in Colorectal Tumorigenesis. Cancer Sci (2017) 108(6):1135-43. doi: $10.1111 /$ cas.13234

84. Miyazawa M, Bogdan AR, Tsuji Y. Perturbation of Iron Metabolism by Cisplatin Through Inhibition of Iron Regulatory Protein 2. Cell Chem Biol (2019) 26(1):85-97.e4. doi: 10.1016/j.chembiol.2018.10.009

85. Yao F, Cui X, Zhang Y, Bei Z, Wang H, Zhao D, et al. Iron Regulatory Protein 1 Promotes Ferroptosis by Sustaining Cellular Iron Homeostasis in Melanoma. Oncol Lett (2021) 22(3):657. doi: 10.3892/ol.2021.12918

86. Pan X, Lu Y, Cheng X, Wang J. Hepcidin and Ferroportin Expression in Breast Cancer Tissue and Serum and Their Relationship With Anemia. Curr Oncol (Toronto Ont) (2016) 23(1):e24-6. doi: 10.3747/co.23.2840

87. Babu KR, Muckenthaler MU. miR-20a Regulates Expression of the Iron Exporter Ferroportin in Lung Cancer. J Mol Med (Berlin Germany) (2016) 94 (3):347-59. doi: 10.1007/s00109-015-1362-3

88. Guo W, Zhang S, Chen Y, Zhang D, Yuan L, Cong H, et al. An Important Role of the Hepcidin-Ferroportin Signaling in Affecting Tumor Growth and Metastasis. Acta Biochim Biophys Sin (2015) 47(9):703-15. doi: 10.1093/ abbs/gmv063

89. Shan Z, Wei Z, Shaikh ZA. Suppression of Ferroportin Expression by Cadmium Stimulates Proliferation, EMT, and Migration in TripleNegative Breast Cancer Cells. Toxicol Appl Pharmacol (2018) 356:36-43. doi: 10.1016/j.taap.2018.07.017

90. Gasparetto M, Pei S, Minhajuddin M, Stevens B, Smith CA, Seligman P. Low Ferroportin Expression in AML Is Correlated With Good Risk Cytogenetics, Improved Outcomes and Increased Sensitivity to Chemotherapy. Leukemia Res (2019) 80:1-10. doi: 10.1016/j.leukres.2019.02.011

91. Jiang X, Zhang C, Qi S, Guo S, Chen Y, Du E, et al. Elevated Expression of ZNF217 Promotes Prostate Cancer Growth by Restraining FerroportinConducted Iron Egress. Oncotarget (2016) 7(51):84893-906. doi: 10.18632/ oncotarget.12753

92. Xue D, Zhou C, Shi Y, Lu H, Xu R, He X. Nuclear Transcription Factor Nrf2 Suppresses Prostate Cancer Cells Growth and Migration Through Upregulating Ferroportin. Oncotarget (2016) 7(48):78804-12. doi: 10.18632/ oncotarget.12860

93. Geng N, Shi BJ, Li SL, Zhong ZY, Li YC, Xua WL, et al. Knockdown of Ferroportin Accelerates Erastin-Induced Ferroptosis in Neuroblastoma Cells. Eur Rev Med Pharmacol Sci (2018) 22(12):3826-36. doi: 10.26355/ eurrev_201806_15267

94. Tang Z, Jiang W, Mao M, Zhao J, Chen J, Cheng N. Deubiquitinase USP35 Modulates Ferroptosis in Lung Cancer via Targeting Ferroportin. Clin Trans Med (2021) 11(4):e390. doi: 10.1002/ctm2.390

95. Mertens C, Schnetz M, Rehwald C, Grein S, Elwakeel E, Weigert A, et al. Iron-Bound Lipocalin-2 From Tumor-Associated Macrophages Drives Breast Cancer Progression Independent of Ferroportin. Metabolites (2021) 11(3):108. doi: 10.3390/metabo11030180

96. Schwartz AJ, Goyert JW, Solanki S, Kerk SA, Chen B, Castillo C, et al. Hepcidin Sequesters Iron to Sustain Nucleotide Metabolism and Mitochondrial Function in Colorectal Cancer Epithelial Cells. Nat Metab (2021) 3(7):969-82. doi: 10.1038/s42255-021-00406-7

97. Abdel-Razeq H, Hashem H. Recent Update in the Pathogenesis and Treatment of Chemotherapy and Cancer Induced Anemia. Crit Rev Oncol/Hematol (2020) 145:102837. doi: 10.1016/j.critrevonc.2019.102837 
98. Manz DH, Blanchette NL, Paul BT, Torti FM, Torti SV. Iron and Cancer: Recent Insights. Ann New York Acad Sci (2016) 1368(1):149-61. doi: 10.1111/nyas.13008

99. Jakszyn P, Fonseca-Nunes A, Lujan-Barroso L, Aranda N, Tous M, Arija V, et al. Hepcidin Levels and Gastric Cancer Risk in the EPIC-EurGast Study. Int J Cancer (2017) 141(5):945-51. doi: 10.1002/ijc.30797

100. Oh ST, Talpaz M, Gerds AT, Gupta V, Verstovsek S, Mesa R, et al. ACVR1/ JAK1/JAK2 Inhibitor Momelotinib Reverses Transfusion Dependency and Suppresses Hepcidin in Myelofibrosis Phase 2 Trial. Blood Adv (2020) 4 (18):4282-91. doi: 10.1182/bloodadvances.2020002662

101. Tseng HH, Chang JG, Hwang YH, Yeh KT, Chen YL, Yu HS. Expression of Hepcidin and Other Iron-Regulatory Genes in Human Hepatocellular Carcinoma and Its Clinical Implications. J Cancer Res Clin Oncol (2009) 135(10):1413-20. doi: 10.1007/s00432-009-0585-5

102. Kijima H, Sawada T, Tomosugi N, Kubota K. Expression of Hepcidin mRNA Is Uniformly Suppressed in Hepatocellular Carcinoma. BMC Cancer (2008) 8:167. doi: 10.1186/1471-2407-8-167

103. Tsiftsoglou AS, Tsamadou AI, Papadopoulou LC. Heme as Key Regulator of Major Mammalian Cellular Functions: Molecular, Cellular, and Pharmacological Aspects. Pharmacol Ther (2006) 111(2):327-45. doi: 10.1016/j.pharmthera.2005.10.017

104. Kalainayakan SP, FitzGerald KE, Konduri PC, Vidal C, Zhang L. Essential Roles of Mitochondrial and Heme Function in Lung Cancer Bioenergetics and Tumorigenesis. Cell Biosci (2018) 8:56. doi: 10.1186/s13578-018-0257-8

105. Tracey N, Creedon H, Kemp AJ, Culley J, Muir M, Klinowska T, et al. HO-1 Drives Autophagy as a Mechanism of Resistance Against HER2-Targeted Therapies. Breast Cancer Res Treat (2020) 179(3):543-55. doi: 10.1007/ s10549-019-05489-1

106. Zhang S, Chen Y, Guo W, Yuan L, Zhang D, Xu Y, et al. Disordered Hepcidin-Ferroportin Signaling Promotes Breast Cancer Growth. Cell Signal (2014) 26(11):2539-50. doi: 10.1016/j.cellsig.2014.07.029

107. Martínez VG, Rubio C, Martínez-Fernández M, Segovia C, López-Calderón F, Garín MI, et al. BMP4 Induces M2 Macrophage Polarization and Favors Tumor Progression in Bladder Cancer. Clin Cancer Res (2017) 23(23):738899. doi: 10.1158/1078-0432.CCR-17-1004

108. Dixon SJ, Lemberg KM, Lamprecht MR, Skouta R, Zaitsev EM, Gleason CE, et al. Ferroptosis: An Iron-Dependent Form of Nonapoptotic Cell Death. Cell (2012) 149(5):1060-72. doi: 10.1016/j.cell.2012.03.042

109. Tang D, Chen X, Kang R, Kroemer G. Ferroptosis: Molecular Mechanisms and Health Implications. Cell Res (2021) 31(2):107-25. doi: 10.1038/s41422-020-00441-1

110. Hirschhorn T, Stockwell BR. The Development of the Concept of Ferroptosis. Free Radical Biol Med (2019) 133:130-43. doi: 10.1016/ j.freeradbiomed.2018.09.043

111. Jiang X, Stockwell BR, Conrad M. Ferroptosis: Mechanisms, Biology and Role in Disease. Nat Rev Mol Cell Biol (2021) 22(4):266-82. doi: 10.1038/ s41580-020-00324-8

112. Gao M, Yi J, Zhu J, Minikes AM, Monian P, Thompson CB, et al. Role of Mitochondria in Ferroptosis. Mol Cell (2019) 73(2):354-63.e3. doi: 10.1016/ j.molcel.2018.10.042

113. Li C, Zhang Y, Liu J, Kang R, Klionsky DJ, Tang D. Mitochondrial DNA Stress Triggers Autophagy-Dependent Ferroptotic Death. Autophagy (2021) 17(4):948-60. doi: 10.1080/15548627.2020.1739447

114. Lee H, Zandkarimi F, Zhang Y, Meena JK, Kim J, Zhuang L, et al. EnergyStress-Mediated AMPK Activation Inhibits Ferroptosis. Nat Cell Biol (2020) 22(2):225-34. doi: 10.1038/s41556-020-0461-8

115. Zhou B, Liu J, Kang R, Klionsky DJ, Kroemer G, Tang D. Ferroptosis Is a Type of Autophagy-Dependent Cell Death. Semin Cancer Biol (2020) 66:89100. doi: 10.1016/j.semcancer.2019.03.002

116. Chen X, Yu C, Kang R, Kroemer G, Tang D. Cellular Degradation Systems in Ferroptosis. Cell Death Differ (2021) 28(4):1135-48. doi: 10.1038/s41418-020-00728-1

117. Mou Y, Wang J, Wu J, He D, Zhang C, Duan C, et al. Ferroptosis, a New Form of Cell Death: Opportunities and Challenges in Cancer. J Hematol Oncol (2019) 12(1):34. doi: 10.1186/s13045-019-0720-y

118. Friedmann Angeli JP, Krysko DV, Conrad M. Ferroptosis at the Crossroads of Cancer-Acquired Drug Resistance and Immune Evasion. Nat Rev Cancer (2019) 19(7):405-14. doi: 10.1038/s41568-019-0149-1

119. Lill R, Mühlenhoff U. Maturation of Iron-Sulfur Proteins in Eukaryotes: Mechanisms, Connected Processes, and Diseases. Annu Rev Biochem (2008) 77:669-700. doi: 10.1146/annurev.biochem.76.052705.162653
120. Terzi EM, Sviderskiy VO, Alvarez SW, Whiten GC, Possemato R. IronSulfur Cluster Deficiency can be Sensed by IRP2 and Regulates Iron Homeostasis and Sensitivity to Ferroptosis Independent of IRP1 and FBXL5. Sci Adv (2021) 7(22):eabg430. doi: 10.1126/sciadv.abg4302

121. Alvarez SW, Sviderskiy VO, Terzi EM, Papagiannakopoulos T, Moreira AL, Adams S, et al. NFS1 Undergoes Positive Selection in Lung Tumours and Protects Cells From Ferroptosis. Nature (2017) 551(7682):639-43. doi: 10.1038/nature24637

122. Rouault TA. Biogenesis of Iron-Sulfur Clusters in Mammalian Cells: New Insights and Relevance to Human Disease. Dis Models Mech (2012) 5 (2):155-64. doi: 10.1242/dmm.009019

123. Du J, Zhou Y, Li Y, Xia J, Chen Y, Chen S, et al. Identification of Frataxin as a Regulator of Ferroptosis. Redox Biol (2020) 32:101483. doi: 10.1016/ j.redox.2020.101483

124. Du J, Wang T, Li Y, Zhou Y, Wang X, Yu X, et al. DHA Inhibits Proliferation and Induces Ferroptosis of Leukemia Cells Through Autophagy Dependent Degradation of Ferritin. Free Radical Biol Med (2019) 131:356-69. doi: 10.1016/j.freeradbiomed.2018.12.011

125. Patra S, Barondeau DP. Mechanism of Activation of the Human Cysteine Desulfurase Complex by Frataxin. Proc Natl Acad Sci USA (2019) 116 (39):19421-30. doi: 10.1073/pnas.1909535116

126. Chafe SC, Vizeacoumar FS, Venkateswaran G, Nemirovsky O, Awrey S, Brown WS, et al. Genome-Wide Synthetic Lethal Screen Unveils Novel CAIX-NFS1/xCT Axis as a Targetable Vulnerability in Hypoxic Solid Tumors. Sci $A d v$ (2021) 7(35):eabj0364. doi: 10.1126/sciadv.abj0364

127. Mao C, Liu X, Zhang Y, Lei G, Yan Y, Lee H, et al. DHODH-Mediated Ferroptosis Defence Is a Targetable Vulnerability in Cancer. Nature (2021) 593(7860):586-90. doi: 10.1038/s41586-021-03539-7

128. Ding Y, Chen X, Liu C, Ge W, Wang Q, Hao X, et al. Identification of a Small Molecule as Inducer of Ferroptosis and Apoptosis Through Ubiquitination of GPX4 in Triple Negative Breast Cancer Cells. J Hematol Oncol (2021) 14 (1):19. doi: 10.1186/s13045-020-01016-8

129. Chen D, Chu B, Yang X, Liu Z, Jin Y, Kon N, et al. Ipla2 $\beta$-Mediated Lipid Detoxification Controls P53-Driven Ferroptosis Independent of GPX4. Nat Commun (2021) 12(1):3644. doi: 10.1038/s41467-021-23902-6

130. Wang X, Chen Y, Wang X, Tian H, Wang Y, Jin J, et al. Stem Cell Factor SOX2 Confers Ferroptosis Resistance in Lung Cancer via Upregulation of SLC7A11. Cancer Res (2021) 81(20):5217-29. doi: 10.1158/0008-5472.CAN21-0567

131. Bao Z, Hua L, Ye Y, Wang D, Li C, Xie Q, et al. MEF2C Silencing Downregulates NF2 and E-Cadherin and Enhances Erastin-Induced Ferroptosis in Meningioma. Neuro-oncology (2021), noab114. doi: 10.1093/ neuonc/noab114

132. Kremer DM, Nelson BS, Lin L, Yarosz EL, Halbrook CJ, Kerk SA, et al. GOT1 Inhibition Promotes Pancreatic Cancer Cell Death by Ferroptosis. Nat Commun (2021) 12(1):4860. doi: 10.1038/s41467-021-24859-2

133. Wang W, Green M, Choi JE, Gijón M, Kennedy PD, Johnson JK, et al. CD8 (+) T Cells Regulate Tumour Ferroptosis During Cancer Immunotherapy. Nature (2019) 569(7755):270-4. doi: 10.1038/s41586-019-1170-y

134. Ye Z, Zhuo Q, Hu Q, Xu X, Mengqi L, Zhang Z, et al. FBW7-NRA41-SCD1 Axis Synchronously Regulates Apoptosis and Ferroptosis in Pancreatic Cancer Cells. Redox Biol (2021) 38:101807. doi: 10.1016/j.redox.2020.101807

135. Hong $\mathrm{T}$, Lei $\mathrm{G}$, Chen $\mathrm{X}$, Li H, Zhang $\mathrm{X}$, Wu N, et al. PARP Inhibition Promotes Ferroptosis via Repressing SLC7A11 and Synergizes With Ferroptosis Inducers in BRCA-Proficient Ovarian Cancer. Redox Biol (2021) 42:101928. doi: 10.1016/j.redox.2021.101928

136. Sun J, Zhou C, Zhao Y, Zhang X, Chen W, Zhou Q, et al. Quiescin Sulfhydryl Oxidase 1 Promotes Sorafenib-Induced Ferroptosis in Hepatocellular Carcinoma by Driving EGFR Endosomal Trafficking and Inhibiting NRF2 Activation. Redox Biol (2021) 41:101942. doi: 10.1016/ j.redox.2021.101942

137. Tan SK, Mahmud I, Fontanesi F, Puchowicz M, Neumann CKA, Griswold AJ, et al. Obesity-Dependent Adipokine Chemerin Suppresses Fatty Acid Oxidation to Confer Ferroptosis Resistance. Cancer Discovery (2021) 11 (8):2072-93. doi: 10.1158/2159-8290.CD-20-1453

138. Lange M, Olzmann JA. Ending on a Sour Note: Lipids Orchestrate Ferroptosis in Cancer. Cell Metab (2021) 33(8):1507-9. doi: 10.1016/ j.cmet.2021.07.011 
139. Dierge E, Debock E, Guilbaud C, Corbet C, Mignolet E, Mignard L, et al. Peroxidation of N-3 and N-6 Polyunsaturated Fatty Acids in the Acidic Tumor Environment Leads to Ferroptosis-Mediated Anticancer Effects. Cell Metab (2021) 33(8):1701-15.e5. doi: 10.1016/j.cmet.2021.05.016

140. Liu W, Chakraborty B, Safi R, Kazmin D, Chang CY, McDonnell DP. Dysregulated Cholesterol Homeostasis Results in Resistance to Ferroptosis Increasing Tumorigenicity and Metastasis in Cancer. Nat Commun (2021) 12(1):5103.doi: 10.1038/s41467-021-25354-4

141. Beatty A, Singh T, Tyurina YY, Tyurin VA, Samovich S, Nicolas E, et al. Ferroptotic Cell Death Triggered by Conjugated Linolenic Acids Is Mediated by ACSL1. Nat Commun (2021) 12(1):2244. doi: 10.1038/s41467-021-22471-y

142. Wang ZX, Ma J, Li XY, Wu Y, Shi H, Chen Y, et al. Quercetin Induces P53Independent Cancer Cell Death Through Lysosome Activation by the Transcription Factor EB and Reactive Oxygen Species-Dependent Ferroptosis. Br J Pharmacol (2021) 178(5):1133-48. doi: 10.1111/bph.15350

143. Wu CY, Yang YH, Lin YS, Chang GH, Tsai MS, Hsu CM, et al. Dihydroisotanshinone I Induced Ferroptosis and Apoptosis of Lung Cancer Cells. BioMed Pharmacother (2021) 139:111585. doi: 10.1016/ j.biopha.2021.111585

144. Wen Y, Chen H, Zhang L, Wu M, Zhang F, Yang D, et al. Glycyrrhetinic Acid Induces Oxidative/Nitrative Stress and Drives Ferroptosis Through Activating NADPH Oxidases and iNOS, and Depriving Glutathione in Triple-Negative Breast Cancer Cells. Free Radical Biol Med (2021) 173:4151. doi: 10.1016/j.freeradbiomed.2021.07.019

145. Mancias JD, Wang X, Gygi SP, Harper JW, Kimmelman AC. Quantitative Proteomics Identifies NCOA4 as the Cargo Receptor Mediating Ferritinophagy. Nature (2014) 509(7498):105-9. doi: 10.1038/nature13148

146. Tang M, Chen Z, Wu D, Chen L. Ferritinophagy/ferroptosis: Iron-Related Newcomers in Human Diseases. J Cell Physiol (2018) 233(12):9179-90. doi: $10.1002 /$ jcp. 26954

147. Ajoolabady A, Aslkhodapasandhokmabad H, Libby P, Tuomilehto J, Lip GYH, Penninger JM, et al. Ferritinophagy and Ferroptosis in the Management of Metabolic Diseases. Trends Endocrinol Metabol: TEM (2021) 32(7):444-62. doi: 10.1016/j.tem.2021.04.010

148. Finazzi D, Arosio P. Biology of Ferritin in Mammals: An Update on Iron Storage, Oxidative Damage and Neurodegeneration. Arch Toxicol (2014) 88 (10):1787-802. doi: 10.1007/s00204-014-1329-0

149. Zarjou A, Jeney V, Arosio P, Poli M, Zavaczki E, Balla G, et al. Ferritin Ferroxidase Activity: A Potent Inhibitor of Osteogenesis. J Bone Mineral Res (2010) 25(1):164-72. doi: 10.1359/jbmr.091002

150. Timoshnikov VA, Kobzeva TV, Polyakov NE, Kontoghiorghes GJ. Inhibition of $\mathrm{Fe}(2+)$ - and $\mathrm{Fe}(3+)$ - Induced Hydroxyl Radical Production by the IronChelating Drug Deferiprone. Free Radical Biol Med (2015) 78:118-22. doi: 10.1016/j.freeradbiomed.2014.10.513

151. Philpott CC. The Flux of Iron Through Ferritin in Erythrocyte Development. Curr Opin Hematol (2018) 25(3):183-8. doi: 10.1097/MOH. 0000000000000417

152. Dowdle WE, Nyfeler B, Nagel J, Elling RA, Liu S, Triantafellow E, et al. Selective VPS34 Inhibitor Blocks Autophagy and Uncovers a Role for NCOA4 in Ferritin Degradation and Iron Homeostasis In Vivo. Nat Cell Biol (2014) 16(11):1069-79. doi: 10.1038/ncb3053

153. Mancias JD, Pontano Vaites L, Nissim S, Biancur DE, Kim AJ, Wang X, et al. Ferritinophagy via NCOA4 Is Required for Erythropoiesis and Is Regulated by Iron Dependent HERC2-Mediated Proteolysis. eLife (2015) 4:e10308. doi: $10.7554 /$ eLife.10308

154. Bellelli R, Federico G, Matte A, Colecchia D, Iolascon A, Chiariello M, et al. NCOA4 Deficiency Impairs Systemic Iron Homeostasis. Cell Rep (2016) 14 (3):411-21. doi: 10.1016/j.celrep.2015.12.065

155. Asano T, Komatsu M, Yamaguchi-Iwai Y, Ishikawa F, Mizushima N, Iwai K. Distinct Mechanisms of Ferritin Delivery to Lysosomes in Iron-Depleted and Iron-Replete Cells. Mol Cell Biol (2011) 31(10):2040-52. doi: 10.1128/ MCB.01437-10

156. Bai Y, Meng L, Han L, Jia Y, Zhao Y, Gao H, et al. Lipid Storage and Lipophagy Regulates Ferroptosis. Biochem Biophys Res Commun (2019) 508 (4):997-1003. doi: 10.1016/j.bbrc.2018.12.039

157. Yang M, Chen P, Liu J, Zhu S, Kroemer G, Klionsky DJ, et al. Clockophagy is a Novel Selective Autophagy Process Favoring Ferroptosis. Sci Adv (2019) 5 (7):eaaw2238. doi: 10.1126/sciadv.aaw2238
158. Liu NQ, De Marchi T, Timmermans AM, Beekhof R, Trapman-Jansen AM, Foekens R, et al. Ferritin Heavy Chain in Triple Negative Breast Cancer: A Favorable Prognostic Marker That Relates to a Cluster of Differentiation 8 Positive (CD8+) Effector T-Cell Response. Mol Cell Proteomics MCP (2014) 13(7):1814-27. doi: 10.1074/mcp.M113.037176

159. Funauchi Y, Tanikawa C, Yi Lo PH, Mori J, Daigo Y, Takano A, et al. Regulation of Iron Homeostasis by the P53-ISCU Pathway. Sci Rep (2015) 5:16497. doi: 10.1038/srep 16497

160. Wu X, Chen F, Sahin A, Albarracin C, Pei Z, Zou X, et al. Distinct Function of Androgen Receptor Coactivator ARA70 $\alpha$ and ARA70 $\beta$ in Mammary Gland Development, and in Breast Cancer. Breast Cancer Res Treat (2011) 128(2):391-400. doi: 10.1007/s10549-010-1131-5

161. Chan JJ, Kwok ZH, Chew XH, Zhang B, Liu C, Soong TW, et al. A FTH1 Gene:Pseudogene:microRNA Network Regulates Tumorigenesis in Prostate Cancer. Nucleic Acids Res (2018) 46(4):1998-2011. doi: 10.1093/nar/gkx1248

162. Yang ND, Tan SH, Ng S, Shi Y, Zhou J, Tan KS, et al. Artesunate Induces Cell Death in Human Cancer Cells via Enhancing Lysosomal Function and Lysosomal Degradation of Ferritin. J Biol Chem (2014) 289(48):33425-41. doi: 10.1074/jbc.M114.564567

163. Xu Z, Feng J, Li Y, Guan D, Chen H, Zhai X, et al. The Vicious Cycle Between Ferritinophagy and ROS Production Triggered EMT Inhibition of Gastric Cancer Cells was Through P53/AKT/mTor Pathway. Chemico-biol Interact (2020) 328:109196. doi: 10.1016/j.cbi.2020.109196

164. Santana-Codina N, Mancias JD. The Role of NCOA4-Mediated Ferritinophagy in Health and Disease. Pharm (Basel Switzerland) (2018) 11(4):114. doi: 10.3390/ph11040114

165. Mou Y, Wu J, Zhang Y, Abdihamid O, Duan C, Li B. Low Expression of Ferritinophagy-Related NCOA4 Gene in Relation to Unfavorable Outcome and Defective Immune Cells Infiltration in Clear Cell Renal Carcinoma. BMC Cancer (2021) 21(1):18. doi: 10.1186/s12885-020-07726-Z

166. Chang K, Yuan C, Liu X. Ferroptosis-Related Gene Signature Accurately Predicts Survival Outcomes in Patients With Clear-Cell Renal Cell Carcinoma. Front Oncol (2021) 11:649347. doi: 10.3389/fonc.2021.649347

167. Zhang Y, Kong Y, Ma Y, Ni S, Wikerholmen T, Xi K, et al. Loss of COPZ1 Induces NCOA4 Mediated Autophagy and Ferroptosis in Glioblastoma Cell Lines. Oncogene (2021) 40(8):1425-39. doi: 10.1038/s41388-020-01622-3

168. Vara-Pérez M, Rossi M, Van den Haute C, Maes H, Sassano ML, Venkataramani V, et al. BNIP3 Promotes HIF-1 $\alpha$-Driven Melanoma Growth by Curbing Intracellular Iron Homeostasis. EMBO J (2021) 40 (10):e106214. doi: 10.15252/embj.2020106214

169. Hasan M, Reddy SM, Das NK. Ferritinophagy is Not Required for Colon Cancer Cell Growth. Cell Biol Int (2020) 44(11):2307-14. doi: 10.1002/ cbin.11439

170. Hayashima K, Kimura I, Katoh H. Role of Ferritinophagy in Cystine Deprivation-Induced Cell Death in Glioblastoma Cells. Biochem Biophys Res Commun (2021) 539:56-63. doi: 10.1016/j.bbrc.2020.12.075

171. Lu R, Jiang Y, Lai X, Liu S, Sun L, Zhou ZW. A Shortage of FTH Induces ROS and Sensitizes RAS-Proficient Neuroblastoma N2A Cells to Ferroptosis. Int $J$ Mol Sci (2021) 22(16):8898. doi: 10.3390/ijms22168898

172. Marcus DM, Zinberg N. Measurement of Serum Ferritin by Radioimmunoassay: Results in Normal Individuals and Patients With Breast Cancer. J Natl Cancer Inst (1975) 55(4):791-5. doi: 10.1093/jnci/ 55.4.791

173. Lee S, Eo W, Jeon H, Park S, Chae J. Prognostic Significance of Host-Related Biomarkers for Survival in Patients With Advanced Non-Small Cell Lung Cancer. J Cancer (2017) 8(15):2974-83. doi: 10.7150/jca.20866

174. Koyama S, Fujisawa S, Watanabe R, Itabashi M, Ishibashi D, Ishii Y, et al. Serum Ferritin Level Is a Prognostic Marker in Patients With Peripheral T-Cell Lymphoma. Int J Lab Hematol (2017) 39(1):112-7. doi: 10.1111/ijlh.12592

175. Khanna V, Karjodkar F, Robbins S, Behl M, Arya S, Tripathi A. Estimation of Serum Ferritin Level in Potentially Malignant Disorders, Oral Squamous Cell Carcinoma, and Treated Cases of Oral Squamous Cell Carcinoma. J Cancer Res Ther (2017) 13(3):550-5. doi: 10.4103/0973-1482.181182

176. Shen Y, Li X, Dong D, Zhang B, Xue Y, Shang P. Transferrin Receptor 1 in Cancer: A New Sight for Cancer Therapy. Am J Cancer Res (2018) 8 (6):916-31.

177. Adachi M, Kai K, Yamaji K, Ide T, Noshiro H, Kawaguchi A, et al. Transferrin Receptor 1 Overexpression Is Associated With Tumour De- 
Differentiation and Acts as a Potential Prognostic Indicator of Hepatocellular Carcinoma. Histopathology (2019) 75(1):63-73. doi: 10.1111/his.13847

178. Liu B, Song Z, Fan Y, Zhang G, Cao P, Li D, et al. Downregulation of FPN1 Acts as a Prognostic Biomarker Associated With Immune Infiltration in Lung Cancer. Aging (2021) 13(6):8737-61. doi: 10.18632/ aging. 202685

179. Cheng X, Fan K, Wang L, Ying X, Sanders AJ, Guo T, et al. TfR1 Binding With H-Ferritin Nanocarrier Achieves Prognostic Diagnosis and Enhances the Therapeutic Efficacy in Clinical Gastric Cancer. Cell Death Dis (2020) 11 (2):92. doi: 10.1038/s41419-020-2272-z

180. Rosager AM, Sørensen MD, Dahlrot RH, Hansen S, Schonberg DL, Rich JN, et al. Transferrin Receptor-1 and Ferritin Heavy and Light Chains in Astrocytic Brain Tumors: Expression and Prognostic Value. PLoS One (2017) 12(8):e0182954. doi: 10.1371/journal.pone.0182954

181. Calzolari A, Larocca LM, Deaglio S, Finisguerra V, Boe A, Raggi C, et al. Transferrin Receptor 2 Is Frequently and Highly Expressed in Glioblastomas. Trans Oncol (2010) 3(2):123-34. doi: 10.1593/tlo.09274

182. Cui C, Cheng X, Yan L, Ding H, Guan X, Zhang W, et al. Downregulation of TfR1 Promotes Progression of Colorectal Cancer via the JAK/STAT Pathway. Cancer Manage Res (2019) 11:6323-41. doi: 10.2147/ CMAR.S198911

183. Zhu B, Zhi Q, Xie Q, Wu X, Gao Y, Chen X, et al. Reduced Expression of Ferroportin1 and Ceruloplasmin Predicts Poor Prognosis in Adrenocortical Carcinoma. J Trace Elements Med Biol (2019) 56:52-9. doi: 10.1016/ j.jtemb.2019.07.009

184. Park CK, Heo J, Ham WS, Choi YD, Shin SJ, Cho NH. Ferroportin and FBXL5 as Prognostic Markers in Advanced Stage Clear Cell Renal Cell Carcinoma. Cancer Res Treat (2021) 53(4):1174-83. doi: 10.4143/ crt.2021.031

185. Wu HT, Chen WJ, Xu Y, Shen JX, Chen WT, Liu J. The Tumor Suppressive Roles and Prognostic Values of STEAP Family Members in Breast Cancer. BioMed Res Int (2020) 2020:9578484. doi: 10.1155/2020/9578484

186. Jerzak KJ, Lohmann AE, Ennis M, Nemeth E, Ganz T, Goodwin PJ. Prognostic Associations of Plasma Hepcidin in Women With Early Breast Cancer. Breast Cancer Res Treat (2020) 184(3):927-35. doi: 10.1007/s10549020-05903-Z

187. Kuang Y, Wang Q. Iron and Lung Cancer. Cancer Lett (2019) 464:56-61. doi: 10.1016/j.canlet.2019.08.007

188. Yu H, Yang C, Jian L, Guo S, Chen R, Li K, et al. Sulfasalazine-induced Ferroptosis in Breast Cancer Cells Is Reduced by the Inhibitory Effect of Estrogen Receptor on the Transferrin Receptor. Oncol Rep (2019) 42(2):82638. doi: 10.3892/or.2019.7189

189. Lelièvre P, Sancey L, Coll JL, Deniaud A, Busser B. Iron Dysregulation in Human Cancer: Altered Metabolism, Biomarkers for Diagnosis, Prognosis, Monitoring and Rationale for Therapy. Cancers (2020) 12(12):3524. doi: $10.3390 /$ cancers 12123524

190. Wang L, Li X, Mu Y, Lu C, Tang S, Lu K, et al. The Iron Chelator Desferrioxamine Synergizes With Chemotherapy for Cancer Treatment. J Trace Elements Med Biol (2019) 56:131-8. doi: 10.1016/j.jtemb.2019.07.008

191. George A, Bobby Z, Dubashi B. Utility of Ferritin and Inflammatory Biomarkers in the Diagnosis of Different Stages of Breast Cancer. Saudi Med J (2021) 42(8):825-31. doi: 10.15537/smj.2021.42.8.20210244

192. Kshattry S, Saha A, Gries P, Tiziani S, Stone E, Georgiou G, et al. EnzymeMediated Depletion of L-Cyst(E)Ine Synergizes With Thioredoxin Reductase Inhibition for Suppression of Pancreatic Tumor Growth. NPJ Precis Oncol (2019) 3:16. doi: 10.1038/s41698-019-0088-z

193. Badgley MA, Kremer DM, Maurer HC, DelGiorno KE, Lee HJ, Purohit V, et al. Cysteine Depletion Induces Pancreatic Tumor Ferroptosis in Mice. Sci (New York NY) (2020) 368(6486):85-9. doi: 10.1126/science.aaw9872

194. Yamasaki T, Terai S, Sakaida I. Deferoxamine for Advanced Hepatocellular Carcinoma. New Engl J Med (2011) 365(6):576-8. doi: 10.1056/ NEJMc1105726

195. Donfrancesco A, Deb G, Dominici C, Pileggi D, Castello MA, Helson L. Effects of a Single Course of Deferoxamine in Neuroblastoma Patients. Cancer Res (1990) 50(16):4929-30.

196. Giles FJ, Fracasso PM, Kantarjian HM, Cortes JE, Brown RA, Verstovsek S, et al. Phase I and Pharmacodynamic Study of Triapine, a Novel
Ribonucleotide Reductase Inhibitor, in Patients With Advanced Leukemia. Leukemia Res (2003) 27(12):1077-83. doi: 10.1016/S0145-2126(03)00118-8

197. Chitambar CR. Gallium Complexes as Anticancer Drugs. Metal ions Life Sci (2018) 18:281-302. doi: 10.1515/9783110470734-010

198. Chitambar CR. The Therapeutic Potential of Iron-Targeting Gallium Compounds in Human Disease: From Basic Research to Clinical Application. Pharmacol Res (2017) 115:56-64. doi: 10.1016/ j.phrs.2016.11.009

199. Najlaoui F, Pigeon P, Aroui S, Pezet M, Sancey L, Marrakchi N, et al. Anticancer Properties of Lipid and Poly(e-Caprolactone) Nanocapsules Loaded With Ferrocenyl-Tamoxifen Derivatives. J Pharm Pharmacol (2018) 70(11):1474-84. doi: 10.1111/jphp.12998

200. Trujillo-Alonso V, Pratt EC, Zong H, Lara-Martinez A, Kaittanis C, Rabie MO, et al. FDA-Approved Ferumoxytol Displays Anti-Leukaemia Efficacy Against Cells With Low Ferroportin Levels. Nat Nanotechnol (2019) 14 (6):616-22. doi: 10.1038/s41565-019-0406-1

201. Ma Y, Chapman J, Levine M, Polireddy K, Drisko J, Chen Q. High-Dose Parenteral Ascorbate Enhanced Chemosensitivity of Ovarian Cancer and Reduced Toxicity of Chemotherapy. Sci Trans Med (2014) 6(222):222ra18. doi: 10.1126/scitranslmed.3007154

202. Welsh JL, Wagner BA, van't Erve TJ, Zehr PS, Berg DJ, Halfdanarson TR, et al. Pharmacological Ascorbate With Gemcitabine for the Control of Metastatic and Node-Positive Pancreatic Cancer (PACMAN): Results From a Phase I Clinical Trial. Cancer Chemother Pharmacol (2013) 71 (3):765-75. doi: 10.1007/s00280-013-2070-8

203. Schoenfeld JD, Sibenaller ZA, Mapuskar KA, Wagner BA, Cramer-Morales KL, Furqan M, et al. $\mathrm{O}(2)(\cdot-)$ and $\mathrm{H}(2) \mathrm{O}(2)$-Mediated Disruption of $\mathrm{Fe}$ Metabolism Causes the Differential Susceptibility of NSCLC and GBM Cancer Cells to Pharmacological Ascorbate. Cancer Cell (2017) 32(2):268. doi: 10.1016/j.ccell.2017.07.008

204. Allen BG, Bodeker KL, Smith MC, Monga V, Sandhu S, Hohl R, et al. FirstIn-Human Phase I Clinical Trial of Pharmacologic Ascorbate Combined With Radiation and Temozolomide for Newly Diagnosed Glioblastoma. Clin Cancer Res (2019) 25(22):6590-7. doi: 10.1158/1078-0432.CCR-19-0594

205. Torti SV, Torti FM. Iron and Cancer: 2020 Vision. Cancer Res (2020) 80 (24):5435-48. doi: 10.1158/0008-5472.CAN-20-2017

206. Mai TT, Hamaï A, Hienzsch A, Cañeque T, Müller S, Wicinski J, et al. Salinomycin Kills Cancer Stem Cells by Sequestering Iron in Lysosomes. Nat Chem (2017) 9(10):1025-33. doi: 10.1038/nchem.2778

207. Liu W, Lin Q, Fu Y, Huang S, Guo C, Li L, et al. Target Delivering Paclitaxel by Ferritin Heavy Chain Nanocages for Glioma Treatment. J Controlled Release (2020) 323:191-202. doi: 10.1016/j.jconrel.2019.12.010

208. Inoue I, Chiba M, Ito K, Okamatsu Y, Suga Y, Kitahara Y, et al. One-Step Construction of Ferritin Encapsulation Drugs for Cancer Chemotherapy. Nanoscale (2021) 13(3):1875-83. doi: 10.1039/D0NR04019C

209. Ni H, Qin H, Sun C, Liu Y, Ruan G, Guo Q, et al. MiR-375 Reduces the Stemness of Gastric Cancer Cells Through Triggering Ferroptosis. Stem Cell Res Ther (2021) 12(1):325. doi: 10.1186/s13287-021-02394-7

210. Wu M, Sun T, Xing L. Circ_0004913 Inhibits Cell Growth, Metastasis, and Glycolysis by Absorbing miR-184 to Regulate HAMP in Hepatocellular Carcinoma. Cancer Biother Radiopharm (2020). doi: 10.1089/cbr.2020.3779

211. Shpyleva SI, Tryndyak VP, Kovalchuk O, Starlard-Davenport A, Chekhun VF, Beland FA, et al. Role of Ferritin Alterations in Human Breast Cancer Cells. Breast Cancer Res Treat (2011) 126(1):63-71. doi: 10.1007/s10549010-0849-4

212. Lui GY, Kovacevic Z, Richardson V, Merlot AM, Kalinowski DS, Richardson DR. Targeting Cancer by Binding Iron: Dissecting Cellular Signaling Pathways. Oncotarget (2015) 6(22):18748-79. doi: 10.18632/oncotarget.4349

213. Steinbrueck A, Sedgwick AC, Brewster JT2nd, Yan KC, Shang Y, Knoll DM, et al. Transition Metal Chelators, Pro-Chelators, and Ionophores as Small Molecule Cancer Chemotherapeutic Agents. Chem Soc Rev (2020) 49 (12):3726-47. doi: 10.1039/C9CS00373H

214. Dreicer R, Kemp JD, Stegink LD, Cardillo T, Davis CS, Forest PK, et al. A Phase II Trial of Deferoxamine in Patients With Hormone-Refractory Metastatic Prostate Cancer. Cancer Invest (1997) 15(4):311-7. doi: 10.3109/07357909709039731

215. Blatt J. Deferoxamine in Children With Recurrent Neuroblastoma. Anticancer Res (1994) 14(5b):2109-12. 
216. Kunos CA, Ivy SP. Triapine Radiochemotherapy in Advanced Stage Cervical Cancer. Front Oncol (2018) 8:149. doi: 10.3389/fonc.2018.00149

217. Merlot AM, Kalinowski DS, Kovacevic Z, Jansson PJ, Sahni S, Huang ML, et al. Exploiting Cancer Metal Metabolism Using Anti-Cancer MetalBinding Agents. Curr Med Chem (2019) 26(2):302-22. doi: 10.2174/ 0929867324666170705120809

218. Knox JJ, Hotte SJ, Kollmannsberger C, Winquist E, Fisher B, Eisenhauer EA. Phase II Study of Triapine in Patients With Metastatic Renal Cell Carcinoma: A Trial of the National Cancer Institute of Canada Clinical Trials Group (NCIC IND.161). Invest New Drugs (2007) 25(5):471-7. doi: 10.1007/ s10637-007-9044-9

219. Nutting CM, van Herpen CM, Miah AB, Bhide SA, Machiels JP, Buter J, et al. Phase II Study of 3-AP Triapine in Patients With Recurrent or Metastatic Head and Neck Squamous Cell Carcinoma. Ann Oncol (2009) 20(7):1275-9. doi: 10.1093/annonc/mdn775

220. Prill S, Rebstock J, Tennemann A, Körfer J, Sönnichsen R, Thieme R, et al. Tumor-Associated Macrophages and Individual ChemoSusceptibility Are Influenced by Iron Chelation in Human Slice Cultures of Gastric Cancer. Oncotarget (2019) 10(46):4731-42. doi: 10.18632/ oncotarget.27089

221. Schnetz M, Meier JK, Rehwald C, Mertens C, Urbschat A, Tomat E, et al. The Disturbed Iron Phenotype of Tumor Cells and Macrophages in Renal Cell Carcinoma Influences Tumor Growth. Cancers (2020) 12(3):530. doi: $10.3390 /$ cancers 12030530

222. Minden MD, Hogge DE, Weir SJ, Kasper J, Webster DA, Patton L, et al. Oral Ciclopirox Olamine Displays Biological Activity in a Phase I Study in Patients With Advanced Hematologic Malignancies. Am J Hematol (2014) 89(4):363-8. doi: 10.1002/ajh.23640

223. Neufeld EJ, Galanello R, Viprakasit V, Aydinok Y, Piga A, Harmatz P, et al. A Phase 2 Study of the Safety, Tolerability, and Pharmacodynamics of FBS0701, A Novel Oral Iron Chelator, in Transfusional Iron Overload. Blood (2012) 119(14):3263-8. doi: 10.1182/blood-2011-10-386268

224. Donfrancesco A, Deb G, Angioni A, Maurizio C, Cozza R, Jenkner A, et al. D-CECaT: A Breakthrough for Patients With Neuroblastoma. Anti-Cancer Drugs (1993) 4(3):317-21. doi: 10.1097/00001813-199306000-00004

225. Shinoda S, Kaino S, Amano S, Harima H, Matsumoto T, Fujisawa K, et al. Deferasirox, an Oral Iron Chelator, With Gemcitabine Synergistically Inhibits Pancreatic Cancer Cell Growth In Vitro and In Vivo. Oncotarget (2018) 9(47):28434-44. doi: 10.18632/oncotarget.25421

226. Zeidner JF, Karp JE, Blackford AL, Smith BD, Gojo I, Gore SD, et al. A Phase II Trial of Sequential Ribonucleotide Reductase Inhibition in Aggressive Myeloproliferative Neoplasms. Haematologica (2014) 99(4):672-8. doi: 10.3324/haematol.2013.097246

227. Straus DJ. Gallium Nitrate in the Treatment of Lymphoma. Semin Oncol (2003) 30(2 Suppl 5):25-33. doi: 10.1016/S0093-7754(03)00173-8
228. Einhorn L. Gallium Nitrate in the Treatment of Bladder Cancer. Semin Oncol (2003) 30(2 Suppl 5):34-41. doi: 10.1016/S0093-7754(03)00174-X

229. Chitambar CR. Gallium Nitrate for the Treatment of Non-Hodgkin's Lymphoma. Expert Opin Invest Drugs (2004) 13(5):531-41. doi: 10.1517/ 13543784.13.5.531

230. Timerbaev AR. Advances in Developing Tris(8-Quinolinolato)Gallium(Iii) as an Anticancer Drug: Critical Appraisal and Prospects. Metallomics Integr Biometal Sci (2009) 1(3):193-8. doi: 10.1039/b902861g

231. Timmers P, Wilson JF, Joshi PK, Deelen J. Multivariate Genomic Scan Implicates Novel Loci and Haem Metabolism in Human Ageing. Nat Commun (2020) 11(1):3570. doi: 10.1038/s41467-020-17312-3

232. Lang X, Green MD, Wang W, Yu J, Choi JE, Jiang L, et al. Radiotherapy and Immunotherapy Promote Tumoral Lipid Oxidation and Ferroptosis via Synergistic Repression of SLC7A11. Cancer Discov (2019) 9(12):1673-85. doi: 10.1158/2159-8290.CD-19-0338

233. Shi Z, Zhang L, Zheng J, Sun H, Shao C. Ferroptosis: Biochemistry and Biology in Cancers. Front Oncol (2021) 11:579286. doi: 10.3389/fonc.2021.579286

234. Yang Y, Li X, Wang T, Guo Q, Xi T, Zheng L. Emerging Agents That Target Signaling Pathways in Cancer Stem Cells. J Hematol Oncol (2020) 13(1):60. doi: 10.1186/s13045-020-00901-6

235. Zhang X, Xing X, Liu H, Feng J, Tian M, Chang S, et al. Ionizing Radiation Induces Ferroptosis in Granulocyte-Macrophage Hematopoietic Progenitor Cells of Murine Bone Marrow. Int J Radiat Biol (2020) 96(5):584-95. doi: 10.1080/09553002.2020.1708993

236. Lei G, Zhang Y, Koppula P, Liu X, Zhang J, Lin SH, et al. The Role of Ferroptosis in Ionizing Radiation-Induced Cell Death and Tumor Suppression. Cell Res (2020) 30(2):146-62. doi: 10.1038/s41422-019-0263-3

Conflict of Interest: The authors declare that the research was conducted in the absence of any commercial or financial relationships that could be construed as a potential conflict of interest.

Publisher's Note: All claims expressed in this article are solely those of the authors and do not necessarily represent those of their affiliated organizations, or those of the publisher, the editors and the reviewers. Any product that may be evaluated in this article, or claim that may be made by its manufacturer, is not guaranteed or endorsed by the publisher.

Copyright (c) $2021 \mathrm{Guo}, \mathrm{Li}$, Hou, Yuan, Li, Zhang, Zheng and Li. This is an openaccess article distributed under the terms of the Creative Commons Attribution License (CC BY). The use, distribution or reproduction in other forums is permitted, provided the original author(s) and the copyright owner(s) are credited and that the original publication in this journal is cited, in accordance with accepted academic practice. No use, distribution or reproduction is permitted which does not comply with these terms. 